Pacific Journal of Mathematics

AN INVESTIGATION OF REAL DIVISION ALGEBRAS USING 


\title{
AN INVESTIGATION OF REAL DIVISION ALGEBRAS USING DERIVATIONS
}

\author{
Georgia M. Benkart and J. Marshall Osborn
}

In a recent paper, "The derivation algebra of a real division algebra", we showed that if $\operatorname{Der} A$ is the derivation algebra of a finite dimensional division algebra $A$ over the reals, then

(i) $\operatorname{dim} A=1$ or 2 implies $\operatorname{Der} A=0$,

(ii) $\operatorname{dim} A=4 \operatorname{implies} \operatorname{Der} A$ is $s u(2)$ or $\operatorname{dim} \operatorname{Der} A=0$ or 1 ,

(iii) $\operatorname{dim} A=8$ implies $\operatorname{Der} A$ is one of the following Lie algebras:

(1) compact $G_{2}$

(2) $s u(3)$

(3) $s u(2) \oplus s u(2)$

(4) $s u(2) \oplus N$ where $N$ is an abelian ideal and $\operatorname{dim} N=0$ or 1

(5) $N$ where $N$ is abelian and $\operatorname{dim} N=0,1$ or 2 .

Moreover, any subalgebra of $\operatorname{Der} A$ is isomorphic to one of the Lie algebras listed above.

For each Lie algebra $L$ appearing in (i), (ii), and (iii) we also exhibited a real division algebra having $L$ as its derivation algebra without proving that the derivation algebra was as asserted. One of the goals of this present paper is to verify that these examples have the derivation algebra claimed, but our main purpose is broader than this. Using the representation theory of Lie algebras we investigate those real division algebras $A$ having $L$ as its derivation algebra for each of the nonzero Lie algebras $L$ mentioned above. The larger that $L$ is, the more detailed is the information concerning the structure of $A$. As one might expect, most of the classes of division algebras are natural generalizations of the quaternions and octonions. The principal exception is a family of division algebras which includes the pseudo-octonions introduced by Okubo in "Pseudo-quaternion and pseudo-octonion algebras."

1. A review of some basic results on representations. Throughout this paper we will assume that all algebras and modules are finite dimensional. Let $A$ be an algebra over a field $F$ of characteristic 0 , and assume $L$ is a semisimple subalgebra of the derivation algebra $\operatorname{Der} A$. Since $A$ is an $L$-module, it decomposes into irreducible summands: $A=V_{1} \oplus \cdots \oplus V_{n}$. Moreover, the product of $V_{r} \times V_{s}$ into $A$ followed by the projection onto $V_{t}$ induces an $L$-module homomorphism of $V_{r} \otimes V_{s}$ into $V_{t}$. Conversely, by taking a sum of irreducible $L$-modules $A=V_{1} \oplus \cdots \oplus V_{n}$ and prescribing 
$L$-module homomorphisms from $V_{r} \otimes V_{s}$ into $V_{t}$ for all $r, s, t$, one achieves an algebra structure on $A$ such that $L \subseteq$ Der $A$. In case $F$ is algebraically closed the dimension of $\operatorname{Hom}_{L}\left(V_{r} \otimes V_{s}, V_{t}\right)$ can be determined using

Proposition 1.1. Let $L$ be a semisimple Lie algebra over an algebraically closed field of characteristic 0. Assume $U$ is an $L$ module and $W$ is an irreducible L-module. If $U=U_{1} \oplus \cdots \oplus U_{m}$ where the $U_{i}$ are irreducible $L$-submodules, then $\operatorname{dim} \operatorname{Hom}_{L}(U, W)$ equals the number of $U_{i}$ isomorphic to $W$.

Since this is a standard result we give only a brief outline of the proof. Using the uniqueness of the decomposition of $U$ and Schur's lemma, one can show that the homomorphisms $\pi_{i}$ (projection of $U$ onto $U_{i}$ followed by an isomorphism onto $W$ ) form a basis for $\operatorname{Hom}_{L}(U, W)$.

In case $U$ is an $L$-module over an arbitrary field $F$ of characteristic 0 , we can take the algebraic closure $K$ of $F$ and form the module $U_{K}=U \otimes_{F} K$ for $L_{K}=L \bigotimes_{F} K$, and then apply Proposition 1.1 to $U_{K}$. We examine the effect of this field extension on certain submodules of $U$.

Suppose $U=U_{1} \oplus \cdots \oplus U_{m}$ is a decomposition of $U$ into irreducible $L$-submodules. Let $U_{0}$ be the sum of all the trivial 1-dimensional summands and $U_{*}$ be the sum of the others. Then $U=U_{0} \oplus$ $U_{*}$ and one readily verifies that:

$$
\begin{aligned}
& U_{0}=\{u \in U \mid l u=0 \text { for all } l \in L\} \\
& U_{*}=L U .
\end{aligned}
$$

The submodules $U_{0}$ and $U_{*}$ behave nicely relative to field extensions as the next lemma indicates.

LEMMA 1.2. (i) $\left(U_{0}\right)_{K}=\left(U_{K}\right)_{0}$

(ii) $\left(U_{*}\right)_{K}=\left(U_{K}\right)_{*}$.

Proof. From our alternate characterizations above, it follows that $\left(U_{0}\right)_{K} \subseteq\left(U_{K}\right)_{0}=\left\{x \in U_{K} \mid l x=0\right.$ for all $\left.l \in L_{K}\right\}$, and $\left(U_{*}\right)_{K}=(L U)_{K} \subseteq$ $L_{K} U_{K}=\left(U_{K}\right)_{*}$. But since $U_{K}=\left(U_{0}\right)_{K} \oplus\left(U_{*}\right)_{K} \subseteq\left(U_{K}\right)_{0} \oplus\left(U_{K}\right)_{*}=U_{K}$, equality must hold in each case.

In view of the above remarks, an equivalent formulation of Lemma 1.2 (ii) is that the extension $(L U)_{K}$ equals the image of $U_{K}$ under $L_{K}$, which is $L_{K} U_{K}$.

We now turn our attention to the case that $A$ is a real division 
algebra. According to the result stated in the introduction, the only possible semisimple subalgebras of Der $A$ are compact $G_{2}, s u(3)$, $s u(2) \oplus s u(2)$, and $s u(2)$. Each of these Lie algebras contains a copy of $s u(2)$ so that if Der $A$ contains a semisimple algebra, $A$ decomposes into irreducible $s u(2)$-modules. Irreducible $s u(2)$-modules are most easily described by complexifying and regarding the resulting module as an $s l(2)$-module. Again the results we mention are quite well-known ([4] or [6]), but our aim is to develop the background needed for later sections.

Let $h=\left(\begin{array}{rr}1 & 0 \\ 0 & -1\end{array}\right), e=\left(\begin{array}{ll}0 & 1 \\ 0 & 0\end{array}\right)$, and $f=\left(\begin{array}{ll}0 & 0 \\ 1 & 0\end{array}\right)$ be the standard basis for $s l(2)$ over the complex numbers $C$. Given any integer $m \geqq 0$, there is a unique irreducible $s l(2)$-module $V(m)$ having dimension $m+1$. We can choose a basis $Z_{m}, Z_{m-2}, \cdots, Z_{m-2 m}=Z_{-m}$ for $V(m)$ so that:

$$
\begin{aligned}
& h Z_{r}=r Z_{r} \\
& e Z_{r}=\frac{m-r}{2} Z_{r+2} \text { where } Z_{m+2}=0 \\
& f Z_{r}=\frac{m+r}{2} Z_{r-2} \text { where } Z_{-m-2}=0 .
\end{aligned}
$$

Now $s u(2)=\left\{x \in \operatorname{sl}(2) \mid \bar{x}^{t}=-x\right\}$, and a basis for $s u(2)$ can be obtained by taking $\partial_{1}=i h, \partial_{2}=e-f$, and $\partial_{3}=i e+i f$. The effect of these elements on the basis of $Z$ 's is given by:

$$
\begin{aligned}
& \partial_{1} Z_{r}=i r Z_{r} \\
& \partial_{2} Z_{r}=\left(\frac{m-r}{2}\right) Z_{r+2}-\left(\frac{m+r}{2}\right) Z_{r-2} \\
& \partial_{3} Z_{r}=i\left(\frac{m-r}{2}\right) Z_{r+2}+i\left(\frac{m+r}{2}\right) Z_{r-2}
\end{aligned}
$$

Let us consider the case that $m=2 n$, and hence that $\operatorname{dim} V(m)=$ $2 n+1$ is odd. In this situation we define:

$$
\begin{aligned}
& U_{q}=Z_{2 q}+(-1)^{q} Z_{-2 q} \text { for } q=0, \cdots, n \\
& V_{q}=i Z_{2 q}-(-1)^{q} i Z_{-2 q} \text { for } q=1, \cdots, n \\
& V_{0}=V_{n+1}=U_{n+1}=0 .
\end{aligned}
$$

The action of $s u(2)$ on the $U$ 's and $V$ 's can be readily computed using (1.4) to show that for $q=1, \cdots, n$ :

$$
\begin{array}{ll}
\partial_{1} U_{q}=2 q V_{q} & \partial_{1} U_{0}=0 \\
\partial_{1} V_{q}=-2 q U_{q} &
\end{array}
$$




$$
\begin{aligned}
& \partial_{2} U_{q}=(n-q) U_{q+1}-(n+q) U_{q-1} \quad \partial_{2} U_{0}=2 n U_{1} \\
& \partial_{2} V_{q}=(n-q) V_{q+1}-(n+q) V_{q-1} \\
& \partial_{3} U_{q}=(n-q) V_{q+1}-(n+q) V_{q-1} \quad \partial_{3} U_{0}=2 n V_{1} \\
& \partial_{3} V_{q}=-(n-q) U_{q+1}-(n+q) U_{q-1} .
\end{aligned}
$$

Thus, if we regard $V(m)$ where $m=2 n$, as a real $s u(2)$-module, the $U$ 's and $V$ 's generate a $s u(2)$-submodule of dimension $m+1$ over $\boldsymbol{R}$, call it $W(m)$. It is not difficult to verify that $W(m)$ is irreducible and that $V(m)=W(m) \oplus i W(m)$ as a real $s u(2)$-module.

The situation when $m$ is odd is completely different. Here $V(m)$ is an irreducible $s u(2)$-module over $\boldsymbol{R}$.

Let us assume $W$ is any irreducible $s u(2)$-module. Then $W_{c}=$ $W \otimes_{R} C$ is an $s l(2)$-module and as such, it decomposes into irreducible submodules of the type $V(m)$. Now $W_{c}$ as an $s u(2)$-module is isomorphic to exactly two copies of $W$. Thus when we regard the $V(m)$ summands as real $s u(2)$-modules we must have a total of two irreducible $s u(2)$-summands each isomorphic to $W$. When $W$ has dimension $2 n+1$ this implies $W$ is isomorphic to $W(2 n)$ and $W_{c} \approx$ $V(2 n)$. If $W$ has dimension $4 n$, then $W_{c} \approx V(2 n-1) \oplus V(2 n-1)$ and $W \approx V(2 n-1)$ when $V(2 n-1)$ is regarded an $s u(2)$-module. There can be no irreducible $s u(2)$-module of dimension $2(2 n+1)$, so in fact, the smallest nontrivial $s u(2)$-module is $s u(2)$ itself.

The Clebsch-Gordan formula provides the answer as to how the tensor product of two irreducible $s l(2)$-modules decomposes:

$$
V(m) \otimes V(n)=V(m+n) \oplus \cdots \oplus V(|m-n|) .
$$

Thus

$$
\begin{aligned}
\operatorname{dim} \operatorname{Hom}_{s l(2)}(V(m) \otimes V(n), V(s)) \\
= \begin{cases}1 & \text { if } s=m+n, m+n-2, \cdots,|m-n| \\
0 & \text { otherwise. }\end{cases}
\end{aligned}
$$

Since for any real Lie algebra $L$ and for any three $L$-modules $U, V, W, \operatorname{dim}_{R} \operatorname{Hom}_{L}(U \otimes V, W) \leqq \operatorname{dim}_{C} \operatorname{Hom}_{L_{C}}\left(U_{C} \otimes V_{C}, W_{c}\right)$, the ClebschGordan formula determines a bound for $\operatorname{dim}_{R} \operatorname{Hom}_{s u(2)}(U \otimes V, W)$.

In addition to results on $s u(2)$ and $s l(2)$-modules we require some facts concerning irreducible modules for $\operatorname{sl}(2) \oplus \operatorname{sl}(2), \operatorname{sl}(3)$, and $G_{2}$. These facts can be established using arguments in ([4], Chapter 6) or ([6], Chapters 7 and 8).

Given a semisimple Lie algebra $L$ over an algebraically closed field of characteristic 0 with Cartan decomposition $L=H \oplus \sum_{\alpha \in \emptyset} L_{\alpha}$, there are certain linear functionals $\lambda_{1}, \cdots, \lambda_{l}$ on $H$, (the so called fundamental weights) which span the dual $H^{*}$ of $H$. The irreducible $L$-modules are in one-to-one correspondence with the elements 
in $H^{*}$ of the form $\lambda=m_{1} \lambda_{1}+\cdots+m_{l} \lambda_{l}$ where the $m_{i}$ are nonnegative integers. Following Humphreys we denote the irreducible module corresponding to $\lambda$ as $V(\lambda)$. (In this notation the $s l(2)$ module $V(m)$ would be $\left.V\left(m \lambda_{1}\right)\right)$. The dimension of the module $V(\lambda)$ is given by Weyl's formula ([4], p. 140), and the tensor product of $V(\lambda)$ and $V\left(\lambda^{\prime}\right)$ can be resolved into irreducibles using either Steinberg's formula ([4], p. 141) or calculations involving weights and their multiplicities.

Real division algebras exist only in dimensions $1,2,4$ and 8, and as the result in the introduction indicates, the only time that $s u(2) \oplus$ $s u(2), s u(3)$, and compact $G_{2}$ occur in $\operatorname{Der} A$ is when $\operatorname{dim} A=8$. Therefore when we decompose $A_{c}$ into irreducible summands for $s l(2) \oplus \operatorname{sl}(2)$, $s l(3)$, or $G_{2}$, the $V(\lambda)$ are constrained by $\operatorname{dim} V(\lambda) \leqq 8$, and the sum of the dimensions must total 8 .

Since every $\operatorname{sl}(2) \oplus s l(2)$ irreducible module is just the tensor product of two irreducible $s l(2)$-modules, one can handle these modules using the above considerations.

For the Lie algebra $s l(3)=A_{2}$, Weyl's dimension formula reads: $\operatorname{dim} V\left(m_{1} \lambda_{1}+m_{2} \lambda_{2}\right)=1 / 2\left(m_{1}+1\right)\left(m_{2}+1\right)\left(m_{1}+m_{2}+2\right)$. Using this expression one easily computes that the only modules of dimension less than 8 are given by

\begin{tabular}{l|c|c} 
& dimension & notation \\
\hline$V(0)$ & 1 & 1 \\
$V\left(\lambda_{1}\right)$ & 3 & 3 \\
$V\left(\lambda_{2}\right)$ & 3 & $\overline{3}$ \\
$V\left(2 \lambda_{1}\right)$ & 6 & 6 \\
$V\left(2 \lambda_{2}\right)$ & 6 & $\overline{6}$ \\
$V\left(\lambda_{1}+\lambda_{2}\right)$ & 8 & 8.
\end{tabular}

We adopt the convention of denoting a module by its dimension, and in the event of two or more of equal dimensions distinguish them by a bar or star or both.

Consider now the tensor products of these modules. For any irreducible $V(\lambda), V(0) \otimes V(\lambda)=V(\lambda)$. Of the remaining products we list only those needed in the study of real division algebras.

$$
\begin{aligned}
& 3 \otimes 3=6+\overline{3} \\
& 3 \otimes \overline{3}=8+1 \\
& \overline{3} \otimes \overline{3}=\overline{6}+3 \\
& 6 \otimes 6=15+15^{*}+\overline{6}
\end{aligned}
$$




$$
\begin{aligned}
& \overline{6} \otimes \overline{6}=\overline{15}+\overline{15}^{*}+6 \\
& 8 \otimes 8=27+10+\overline{10}+8+8+1 .
\end{aligned}
$$

Here $15=V\left(4 \lambda_{1}\right), 15^{*}=V\left(2 \lambda_{1}+\lambda_{2}\right), \overline{15}=V\left(4 \lambda_{2}\right), \overline{15}^{*}=V\left(\lambda_{1}+2 \lambda_{2}\right)$, $27=V\left(2 \lambda_{1}+2 \lambda_{2}\right), 10=V\left(3 \lambda_{1}\right)$ and $\overline{10}=V\left(3 \lambda_{2}\right)$.

The case $8 \otimes 8$ is noteworthy because the two 8 -dimensional summands imply there are two linearly independent $s l(3)$-homomorphisms of $8 \otimes 8 \rightarrow 8$. The 8 -dimensional module is $s l(3)$ itself under the adjoint representation, and a basis of homomorphisms may be concretely given by: $x \otimes y \rightarrow[x y]$ and $x \otimes y \rightarrow x y+y x-2 / 3 \operatorname{tr}(x y) I$ where $\operatorname{tr}(x y)$ denotes the trace of $x y$.

The dimension formula for $G_{2}$ modules is

$\operatorname{dim} V(\lambda)$

$=\frac{1}{5 !}\left(m_{1}+1\right)\left(m_{2}+1\right)\left(m_{1}+m_{2}+2\right)\left(m_{1}+2 m_{2}+3\right)\left(m_{1}+3 m_{2}+4\right)\left(2 m_{1}+3 m_{2}+5\right)$.

Thus, there are only two modules of dimension less than or equal to 8: the 1-dimensional module $V(0)$, and the 7-dimensional module $V\left(\lambda_{1}\right)$. The resolution of $7 \otimes 7$ into irreducibles is given by

$$
7 \otimes 7=V\left(2 \lambda_{1}\right) \oplus V\left(\lambda_{2}\right) \oplus V\left(\lambda_{1}\right) \oplus V(0)
$$

where these modules have dimensions $27,14,7$, and 1 respectively.

2. The case Der $A=$ compact $G_{2}$. We are now ready to consider individually the different possibilities for $\operatorname{Der} A$, and to investivate for each one the division algebras $A$ with that derivation algebra. We take the possible derivation algebras in the order in which they are listed at the beginning of this paper, starting with the case when Der $A$ is a compact form of $G_{2}$. As we noted in $\S 1$, there are only two irreducible $G_{2}$-modules of dimension 8 or less over the complex numbers-one of dimension 1 and one of dimension 7. Thus, if $A$ is a real division algebra with $\operatorname{Der} A=$ compact $G_{2}$, the scalar extension $A_{c}=A \otimes_{R} C$ must be a sum of one 1-dimensional module and one 7-dimensional module. (Note $A_{c}$ could not be a sum of eight 1-dimensional modules because $\operatorname{Der} A$ must act faithfully on $A$.) Since the decomposition of $A_{c}$ into irreducible modules is necessarily a refinement of the decomposition of $A$, we see that either $A$ is a direct sum of a 1-dimensional module and an irreducible 7-dimensional module, or else $A$ is an irreducible 8-dimensional module. But the last possibility can be ruled out by Lemma 1.2. Hence $A=U+V$ where $U$ is a 1-dimensional $G_{2}$-module and $V$ is an irreducible 7-dimensional $G_{2}$-module.

As was observed at the beginning of $\S 1$, the homomorphisms 
from $U \otimes U, \quad U \otimes V, \quad V \otimes U$, and $V \otimes V$ into $U$ and $V$ determine the possible products between the summands. Since for $G_{2}$-modules over $C, 1 \otimes 1 \cong 1,1 \otimes 7 \cong 7 \cong 7 \otimes 1$, and $7 \otimes 7 \cong 27+$ $14+7+1$, it follows from Proposition 1.1 that there is at most one homomorphism up to scalar multiple in each of the cases: $U \otimes$ $U \rightarrow U, \quad U \otimes V \rightarrow V, \quad V \otimes U \rightarrow V, \quad V \otimes V \rightarrow V$, and $V \otimes V \rightarrow U$, and only the zero homomorphism in the other cases. From this we deduce first that $U^{2} \cong U$. But since $A$ is a division algebra, $U^{2} \neq 0$, so it must be $U^{2}=U$. Thus, there exists an idempotent $u \in U$. Now $u \otimes v \rightarrow v$ and $v \otimes u \rightarrow v$ define module homomorphisms from $U \otimes V$ and $V \otimes U$ onto $V$. Therefore, left (right) multiplication by $u$ is just the identity transformation on $V$ multiplied by the scalar $\eta(\zeta)$. To determine homomorphisms for $V \otimes V \rightarrow V, V \otimes$ $V \rightarrow U$, we examine the best known example in the class we are describing - the octonion algebra $O$. In $O$ there is a basis $u, e_{1}, \cdots, e_{7}$ with multiplication given by table (2.1) below with $\beta=\eta=\zeta=1$. Here $u$ spans a 1 -dimensional module and $e_{1}, \cdots, e_{7}$ a 7 -dimensional module for Der $\boldsymbol{O}=$ compact $G_{2}$. Since the modules being discussed are unique up to isomorphism, and since $\operatorname{dim}_{R} \operatorname{Hom}_{G_{2}}(V \otimes V, V) \leqq 1$ and $\operatorname{dim}_{R} \operatorname{Hom}_{G_{2}}(V \otimes V, U) \leqq 1$, the products in the general case are the same as in the octonions up to multiplication by a constant. After replacing the basis elements of $V$ by a fixed scalar multiple of themselves, we may assume that the multiplication from $V \times V$ to $V$ is identical to that of the octonions, but that the products from $V \times V$ to $U$ involve the scalar $\beta$. To be specific, there is a basis $u, e_{1}, \cdots, e_{7}$ with multiplication given by

\begin{tabular}{c|c|rrrrrrr|}
\multicolumn{1}{c}{$u$} & $e_{1}$ & $e_{2}$ & $e_{3}$ & $e_{4}$ & $e_{5}$ & $e_{6}$ & $e_{7}$ \\
\cline { 2 - 8 }$u$ & $u$ & $\eta e_{1}$ & $\eta e_{2}$ & $\eta e_{3}$ & $\eta e_{4}$ & $\eta e_{5}$ & $\eta e_{8}$ & $\eta e_{7}$ \\
\cline { 2 - 8 }$e_{1}$ & $\zeta e_{1}$ & $-\beta u$ & $e_{4}$ & $e_{7}$ & $-e_{2}$ & $e_{6}$ & $-e_{5}$ & $-e_{3}$ \\
$e_{2}$ & $\zeta e_{2}$ & $-e_{4}$ & $-\beta u$ & $e_{5}$ & $e_{1}$ & $-e_{3}$ & $e_{7}$ & $-e_{6}$ \\
$e_{3}$ & $\zeta e_{3}$ & $-e_{7}$ & $-e_{5}$ & $-\beta u$ & $e_{8}$ & $e_{2}$ & $-e_{4}$ & $e_{1}$ \\
$e_{4}$ & $\zeta e_{4}$ & $e_{2}$ & $-e_{1}$ & $-e_{6}$ & $-\beta u$ & $e_{7}$ & $e_{3}$ & $-e_{5}$ \\
$e_{5}$ & $\zeta e_{5}$ & $-e_{6}$ & $e_{3}$ & $-e_{2}$ & $-e_{7}$ & $-\beta u$ & $e_{1}$ & $e_{4}$ \\
$e_{6}$ & $\zeta e_{8}$ & $e_{5}$ & $-e_{7}$ & $e_{4}$ & $-e_{3}$ & $-e_{1}$ & $-\beta u$ & $e_{2}$ \\
$e_{7}$ & $\zeta e_{7}$ & $e_{3}$ & $e_{6}$ & $-e_{1}$ & $e_{5}$ & $-e_{4}$ & $-e_{2}$ & $-\beta u$ \\
\hline
\end{tabular}

The final thing that we wish to determine in this case is for which values of $\beta, \eta, \zeta$ the algebra with the above table is a division algebra. In particular, we shall establish

THEOREM 2.2. A real algebra $A$ is a division algebra with the 
compact form of $G_{2}$ as its derivation algebra if and only if $A$ has $a$ basis $u, e_{1}, \cdots, e_{7}$ with multiplication given by (2.1) for some real numbers $\beta, \eta, \zeta$ such that $\beta \eta \zeta>0$.

Proof. In view of our preceding discussion, it remains only to show that the algebra $A$ whose multiplication table is given by (2.1) is a division algebra exactly when $\beta \eta \zeta>0$. The condition for $A$ to be a division algebra is that the relation

$$
0=\left(a_{0} u+\sum_{i=1}^{7} a_{i} e_{i}\right)\left(b_{0} u+\sum_{i=1}^{7} b_{i} e_{i}\right)
$$

can hold for real $a$ 's and $b$ 's only if either all $a$ 's are zero or all $b$ 's are zero. As in the proof of ([1], Theorem 20), we multiply out the right side of (2.3) and set the coefficients equal to zero. If the $b$ 's are regarded as variables in the resulting equations, the coefficient matrix is

$$
M_{1}=\left(\begin{array}{rrrrrrrr}
a_{0} & -\beta a_{1} & -\beta a_{2} & -\beta a_{3} & -\beta a_{4} & -\beta a_{5} & -\beta a_{6} & -\beta a_{7} \\
\zeta a_{1} & \eta a_{0} & -a_{4} & -a_{7} & a_{2} & -a_{6} & a_{5} & a_{3} \\
\zeta a_{2} & a_{4} & \eta a_{0} & -a_{5} & -a_{1} & a_{3} & -a_{7} & a_{6} \\
\zeta a_{3} & a_{7} & a_{5} & \eta a_{0} & -a_{6} & -a_{2} & a_{4} & -a_{1} \\
\zeta a_{4} & -a_{2} & a_{1} & a_{8} & \eta a_{0} & -a_{7} & -a_{3} & a_{5} \\
\zeta a_{5} & a_{6} & -a_{3} & a_{2} & a_{7} & \eta a_{0} & -a_{1} & -a_{4} \\
\zeta a_{6} & -a_{5} & a_{7} & -a_{4} & a_{3} & a_{1} & \eta a_{0} & -a_{2} \\
\zeta a_{7} & -a_{3} & -a_{6} & a_{1} & -a_{5} & a_{4} & a_{2} & \eta a_{0}
\end{array}\right) .
$$

The statement that $A$ is a division algebra is equivalent to the condition that the determinant of $M_{1}$ is nonzero unless all the $a$ 's are zero. Since $\eta$ and $\zeta$ must be nonzero for $A$ to be a division algebra, we can replace each $a_{0}$ with $\eta^{-1} a_{0}$ and then multiply each entry in the first column by $\zeta^{-1}$. If the resulting matrix is called $M_{2}$, then $\operatorname{det} M_{1}=0$ if and only if $\operatorname{det} M_{2}=0$. Now let us suppose that the matrix $M$ is the same matrix as $M_{2}$ only with $\eta^{-1}=\zeta^{-1}=$ $\beta=1$. Since $M$ corresponds to the octonion algebra, $\operatorname{det} M \neq 0$ unless all $\alpha_{i}$ are 0 . Thus $\operatorname{det} M_{2}=0$ if and only if $\operatorname{det} M_{2} M^{t}=0$. But $M_{2} M^{t}$ has no entries below the diagonal since the last 7 rows of $M_{2}$ and $M$ are the same and are pairwise orthogonal. Hence, the determinant of $M_{2} M^{t}$ is the product of the diagonal elements. The first diagonal entry is $\eta^{-1} \zeta^{-1} a_{0}^{2}+\sum \beta a_{i}^{2}$ and the remaining entries are $a_{0}^{2}+\sum_{i=1}^{7} a_{i}^{2}$. It is clear that if $\eta^{-1} \zeta^{-1}$ and $\beta$ are both positive or both negative, then the determinant of $M_{2} M^{t}$ is not 0 unless all the $a$ 's are, hence $A$ is a division algebra. Conversely, if $A$ is a 
division algebra, $\eta^{-1} \zeta^{-1} a_{0}^{2}+\sum_{i=1}^{7} \beta a_{i}^{2}$ is not zero unless all the $a$ 's vanish, which implies that $\eta^{-1} \zeta^{-1}$ and $\beta$ have the same sign. Thus, $A$ is a division algebra if and only if $\beta \eta \zeta>0$.

3. The case $\operatorname{Der} A=s u(3)$ begun. In this section we investigate the case when $A$ is an 8-dimensional irreducible $s u(3)$-module. Then $A$ is isomorphic to $s u(3)$ when it is regarded as an $s u(3)$ module under the adjoint representation. As we saw in $\S 1$ there are two independent homomorphisms from $s l(3) \otimes s l(3)$ to $s l(3)$, and this is indeed true for $s u(3)$ as well. One of the homomorphisms is obviously the Lie product, and to obtain the other we consider $s u(3)$ as $3 \times 3$ complex skew-Hermitian matrices $\left(\bar{x}^{t}=-x\right)$ of trace zero. For $x$ and $y$ in $s u(3), x y+y x-(2 / 3) \operatorname{tr}(x y) I$ is a Hermitian matrix, so multiplying it by $i$ gives a skew-Hermitian matrix which also has trace zero. Now for $z$ also in $s u(3)$,

$$
\begin{gathered}
{\left[z, i\left\{x y+y x-\frac{2}{3} \operatorname{tr}(x y) I\right\}\right]=i\{[z x] y+y[z x]+x[z y]+[z y] x\}} \\
=i\left\{[z x] y+y[z x]-\frac{2}{3} \operatorname{tr}([z x] y) I\right\} \\
+i\left\{x[z y]+[z y] x-\frac{2}{3} \operatorname{tr}(x[z y]) I\right\}
\end{gathered}
$$

since $\operatorname{tr}([z x] y)=-\operatorname{tr}([x z] y)=-\operatorname{tr}(x[z y])$. This calculation demonstrates that the map $x \otimes y \rightarrow i\{x y+y x-(2 / 3) \operatorname{tr}(x y) I\}$ is indeed an $s u(3)$-homomorphism. Our argument shows that for any real 8-dimensional algebra $A$ on which $s u(3)$ acts irreducibly as derivations, the product in $A$ is given by

$$
x * y=\alpha[x y]+\beta i\left\{x y+y x-\frac{2}{3} \operatorname{tr}(x y) I\right\} .
$$

In fact, $s u(3)$ is the entire derivation algebra whenever $\alpha \neq 0$. For if $A^{-}$denotes the algebra $A$ under the product $x * y-y * x=2 \alpha[x y]$, then every derivation of $A$ is also a derivation of $A^{-}$. But $A^{-}$is isomorphic to $s u(3)$, which has only inner derivations (see [4] p. 23), so Der $A=s u(3)$ in this instance. Our investigations of this case will be complete, once we establish a criterion for such an algebra to be a division algebra. To this purpose we prove

THEOREM 3.2. Let $A$ be an 8-dimensional real algebra defined on the vector space su(3) with multiplication given by (3.1). Then $A$ is a division algebra if and only if $\alpha \beta \neq 0$. For such a division algebra, Der $A=s u(3)$ and $A$ is an irreducible su(3)-module. Conversely any real division algebra on which su(3) acts irreducibly 
as derivations is given by this construction.

It is easy to see the necessity of the condition $\alpha \beta \neq 0$ to have a division algebra since any element squares to zero if $\beta=0$, and since the product of $i e_{11}-i e_{22}$ with $e_{12}-e_{21}$ is zero if $\alpha=0$. To establish the sufficiency of the condition we need the following results.

Let $x$ be a skew-Hermitian complex matrix. Then there is a unitary matrix $u$ such that $u^{-1} x u=v$ where $v$ is diagonal (see for instance, Herstein [3] p. 302, Theorem 6. $Z_{2}$ ). Since $v$ is skewHermitian also, it follows that all the characteristic roots of $v$, hence of $x$, are purely imaginary.

LEMma 3.3. Let $x$ and $y$ belong to su(3), and assume $\gamma, \delta \in C$ are such that $\delta \neq \pm \gamma$. If $\gamma x y+\delta y x=\lambda I$ for some $\lambda \in C$, then $x$ or $y$ is 0 .

Proof. Let $u$ be a unitary matrix which diagonalizes $x$ as above. Then $\gamma\left(u^{-1} x u\right)\left(u^{-1} y u\right)+\delta\left(u^{-1} x u\right)\left(u^{-1} y u\right)=\lambda I$. Hence we may assume without loss of generality that $x$ is diagonal, say $x=$ diag $\left\{a_{1}, a_{2}, a_{3}\right\}$. If $y=\left(b_{i j}\right)$, then the equation $\gamma x y+\delta y x=\lambda I$ gives for $i \neq j$ :

$$
\left(\gamma a_{i}+\delta a_{j}\right) b_{i j}=0
$$

Since $y$ is skew-Hermitian, $b_{j i}=-\bar{b}_{i j}$. Thus, for each pair $i, j$ with $i \neq j$, we obtain the system of equations:

$$
\begin{aligned}
& \left(\gamma a_{i}+\delta a_{j}\right) b_{i j}=0, \\
& \left(\gamma a_{j}+\delta a_{i}\right) \bar{b}_{i j}=0 .
\end{aligned}
$$

If some $b_{i j} \neq 0$ for $i \neq j$, then since $\gamma^{2}-\delta^{2} \neq 0$, we have $a_{i}=a_{j}=0$. However, $x$ has trace 0 , so it follows that $a_{1}=a_{2}=a_{3}=0$ in this case, and $x=0$. We may assume then that $y$ is diagonal, say $y=$ $\operatorname{diag}\left\{b_{1}, b_{2}, b_{3}\right\}$. Equating entries in $\gamma x y+\delta y x=\lambda I$ gives

$$
a_{1} b_{1}=a_{2} b_{2}=a_{3} b_{3}=(\gamma+\delta)^{-1} \lambda \text {. }
$$

Using the fact that $x$ and $y$ have trace 0 , we obtain

$$
a_{1} b_{1}=a_{2} b_{2}=\left(-a_{1}-a_{2}\right)\left(-b_{1}-b_{2}\right)
$$

which simplifies to show:

$$
a_{1} b_{1}-a_{2} b_{2}=0, \quad a_{1}\left(b_{1}+b_{2}\right)+a_{2} b_{1}=0 .
$$


If not both $a_{1}$ and $a_{2}$ are zero then

$$
0=\left|\begin{array}{lr}
b_{1} & -b_{2} \\
b_{1}+b_{2} & b_{1}
\end{array}\right|=b_{1}^{2}+b_{1} b_{2}+b_{2}^{2} .
$$

If $b_{2}=0$, then $b_{1}=0$ and $y=0$ as well. So we may assume $b_{2} \neq 0$. Then it is apparent from (3.8) that $b_{1} b_{2}^{-1}$ satisfies the equation $z^{2}+$ $z+1$. Hence $b_{1} b_{2}^{-1}=\omega$, a complex cube root of 1 . But then (3.5) implies $a_{2}=a_{1} \omega$ and $a_{3}=-a_{1}-a_{2}=-a_{1}(1+\omega)=a_{1} \omega^{2}$. If $a_{1}=\alpha i$ for $\alpha \in \boldsymbol{R}$, then $a_{2}=(\alpha / 2) i \pm \sqrt{(3 / 2)} \alpha$ which contradicts the fact that all roots of $x$ are purely imaginary unless $\alpha=0$. But then $x=0$ as desired.

Proof of Theorem 3.2. It remains to show that if $\alpha \beta \neq 0$ then $A$ is a division algebra. Suppose $x$ and $y$ are complex skewHermitian $3 \times 3$ matrices of trace zero with the property that

$$
\begin{aligned}
0 & =x * y=\alpha[x y]+\beta i\left\{x y+y x-\frac{2}{3} \operatorname{tr}(x y) I\right\} \\
& =(\alpha+\beta i) x y+(-\alpha+\beta i) y x-\frac{2}{3} \beta i \operatorname{tr}(x y) I .
\end{aligned}
$$

Letting $\gamma=\alpha+\beta i$ and $\delta=-\alpha+\beta i$, we have $\gamma+\delta=2 \beta i \neq 0$ and $\gamma-\delta=2 \alpha \neq 0$. Since the hypotheses of Lemma 3.3 are satisfied, we are forced to conclude that $x=0$ or $y=0$, and hence that $A$ is a division algebra.

Those special cases in which $\beta= \pm \sqrt{3 \alpha}$ have been studied recently by Okubo [8], and have been shown to have many interesting properties. For example these algebras have a quadratic form permitting composition. They are not composition algebras in the usual sense since they do not have an identity element.

It turns out that the two algebras studied by Okubo are the only ones in the class defined by Theorem 3.2 which have a quadratic form permitting composition. However, we can show that every algebra $A$ described by Theorem 3.2 is flexible. For this we take $x, y$ skew-Hermitian matrices of trace 0 , we let $\lambda_{x, y}=(2 / 3) \operatorname{tr}(x y)$ and use (3.1) to calculate that

$$
\begin{aligned}
(x * y) & * x-x *(y * x) \\
= & \left(\alpha[x y]+\beta i\left\{x y+y x-\lambda_{x, y} I\right\}\right) * x-x *\left(\alpha[y x]+\beta i\left\{x y+y x-\lambda_{x, y} I\right\}\right) \\
= & \alpha^{2}([[x y] x]-[x[y x]])+\alpha \beta i\left([x y] x+x[x y]-\lambda_{[x y], x} I-x[y x]\right. \\
& \left.-[y x] x+\lambda_{x,[x y]} I+[x y+y x, x]-[x, x y+y x]\right)
\end{aligned}
$$




$$
\begin{aligned}
& -\beta^{2}\left(x y x+y x^{2}+x^{2} y+x y x-2 \lambda_{x, y} x-\lambda_{x y+y x, x} I-x^{2} y-2 x y x\right. \\
& \left.-y x^{2}+2 \lambda_{x, y} x+\lambda_{x, x y+y x} I\right) \\
= & \alpha \beta i(2[x y] x+2 x[x y]+2[x y+y x, x])=0 .
\end{aligned}
$$

It is also clear from (3.1) that $A$ is Lie admissible, since $A^{-} \cong s u(3)$.

4. The case Der $A=s u(3)$ concluded. Having dealt with the situation when $A$ is a single irreducible $s u(3)$-module, we turn to the case when $A$ is a sum of at least two irreducible $s u(3)$-modules. The only irreducible $s l(3)$-modules of dimension less than 8 are the ones which in the notation of (1.7) are given by $1,3, \overline{3}, 6$ and $\overline{6}$. Thus $A_{c}$ must be a sum of modules of these types which add up to give $\operatorname{dim} A_{c}=8$. We consider the various possibilities.

First, if $A_{c}$ consists of a sum of 1's and 3's, then the relation $3 \otimes 3=6+\overline{3}$ in (1.8) shows that the product of any two elements in the 3-summand(s) must be zero. However, by Lemma 1.2 the complexification of the image $(s u(3) A)_{c}$ equals $s l(3) A_{c}$ which is the sum of copies of 3. Thus, the product of any two elements in $s u(3) A$ would be zero and would contradict the fact that $A$ is a division algebra. This demonstrates that $A_{c}$ cannot consist solely of 1 's and 3 's. Similarly we can rule out each case where in addition to 1 's there is exactly one of the types $\overline{3}, 6$, or $\overline{6}$ occurring in $A_{c}$ by using the relations $\overline{3} \otimes \overline{3}=\overline{6}+3,6 \otimes 6=15+15^{*}+\overline{6}$, and $\overline{6} \otimes \overline{6}=$ $\overline{15}+\overline{15}^{*}+6$ from (1.8).

Thus, there must be at least two of the types $3, \overline{3}, 6, \overline{6}$ present in $A_{c}$, and this implies $A_{c}=1+1+3+\overline{3}$. Looking again at $s u(3) A$ and $\operatorname{sl}(3) A_{c}$, we see that $A$ is the sum of two 1-dimensional modules and either two nonisomorphic 3-dimensional irreducible $s u(3)$-modules or one irreducible 6-dimensional module. In the former case let us suppose $W, \bar{W}$ are the two 3 -dimensional modules such that $W_{c}=3$ and $\bar{W}_{c}=\overline{3}$, and $U$ and $V$ are the 1-dimensional modules. Then the relations $3 \otimes 1=3,3 \otimes 3=6+\overline{3}$, and $\overline{3} \otimes 3=8+1$ show that for each $w \in W, w A \subseteq \boldsymbol{R} w+U+V+\bar{W}$. Hence, left multiplication by $w$ is not onto, and this case cannot happen if $A$ is a division algebra. Thus $A$ is the direct sum of two 1-dimensional modules and an irreducible 6-dimensional module $Z$. Moreover we have the following

THEOREM 4.1. If $A$ is a real division algebra such that Der $A=s u(3)$ and $A$ is not an irreducible su(3)-module, then $A$ has $a$ basis $u, v, z_{1}, \cdots, z_{6}$ with multiplication table given by (4.2). Conversely an algebra $A$ defined by (4.2) admits su(3) as derivations. 


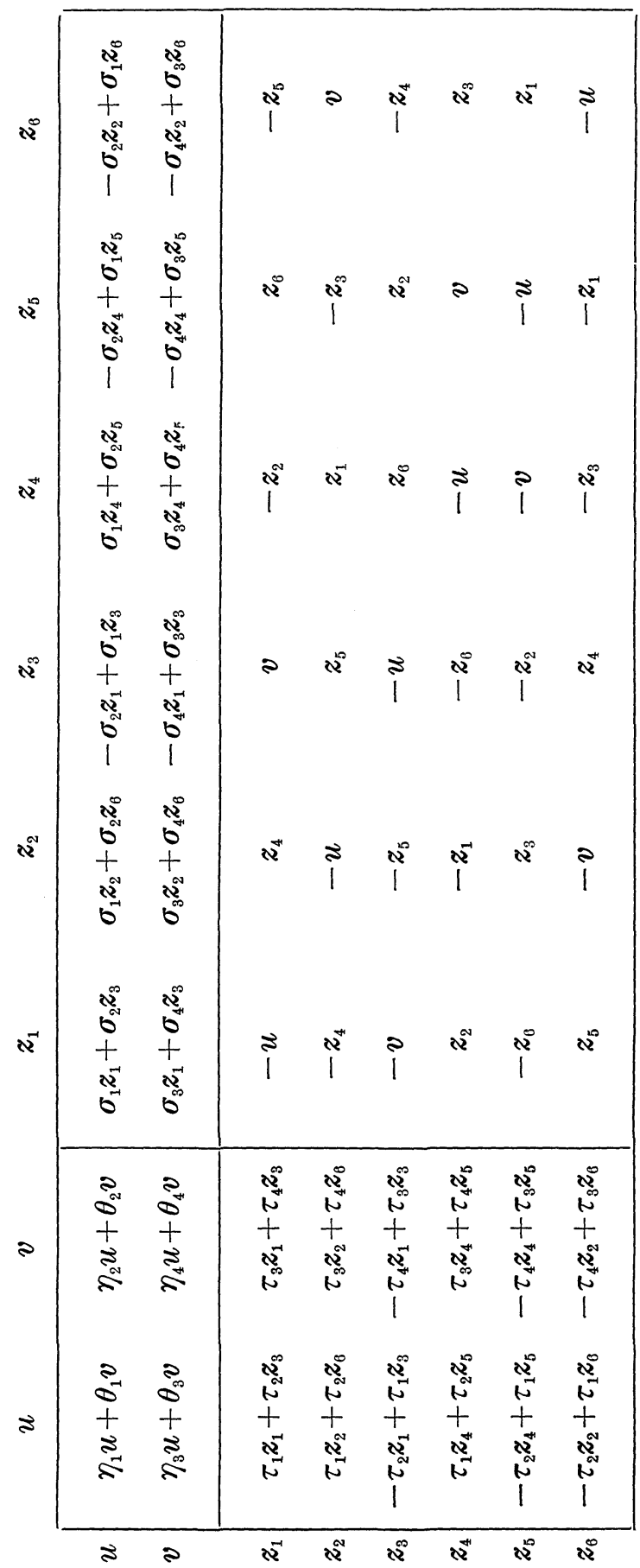


Proof. We have already determined that such an algebra is the sum of two 1-dimensional su(3)-modules and an irreducible 6dimensional module. In order to deduce the various products between the summands let us first consider a well-known example in which this type of decomposition occurs-namely the octonions. Let $O$ be an octonion algebra with basis $u, e_{1}, \cdots, e_{7}$ and multiplication given by (2.1) with $\beta=\zeta=\eta=1$. Let $L=\left\{\partial \in \operatorname{Der} O \mid \partial\left(e_{7}\right)=0\right\}$. Then $L$ is isomorphic to $s u(3)$. (See for example, [2], [5], or [7].) One can actually verify this assertion directly in the following manner. Let us complexify $\boldsymbol{O}$ and obtain a basis for $\boldsymbol{O}_{\boldsymbol{c}}$ by taking:

$$
\begin{array}{ll}
u_{0}=\frac{1}{2}\left(u+i e_{7}\right) & u_{0}^{*}=\frac{1}{2}\left(u-i e_{7}\right) \\
u_{1}=\frac{1}{2}\left(e_{1}+i e_{3}\right) & u_{1}^{*}=\frac{1}{2}\left(e_{1}-i e_{3}\right) \\
u_{2}=\frac{1}{2}\left(e_{2}+i e_{6}\right) & u_{2}^{*}=\frac{1}{2}\left(e_{2}-i e_{6}\right) \\
u_{3}=\frac{1}{2}\left(e_{4}+i e_{5}\right) & u_{3}^{*}=\frac{1}{2}\left(e_{4}-i e_{5}\right) .
\end{array}
$$

Products between these elements can be calculated using (2.1). We list the results below where we adopt the convention that $\varepsilon_{j k l}=1$ if $(j k l)$ is an even permutation of $\{1,2,3\}, \varepsilon_{j k l}=-1$ if the permutation is odd, and $\varepsilon_{j k l}=0$ if $(j k l)$ is not a permutation of $\{1,2,3\}$, and $\delta_{j k}$ is the Kronecker delta.

$$
\begin{array}{llll}
u_{0} u_{j}=u_{j} & u_{0}^{*} u_{j}=0 & u_{j} u_{0}=0 & u_{j} u_{0}^{*}=u_{j} \\
u_{0} u_{j}^{*}=0 & u_{0}^{*} u_{j}^{*}=u_{j}^{*} & u_{j}^{*} u_{0}=u_{j}^{*} & u_{j}^{*} u_{0}^{*}=0 \\
u_{0}^{2}=u_{0} & u_{0} u_{0}^{*}=0=u_{0}^{*} u_{0} & \left(u_{0}^{*}\right)^{2}=u_{0}^{*} \\
u_{j} u_{k}=\varepsilon_{j k l} u_{l}^{*} & u_{j} u_{k}^{*}=-\delta_{j k} u_{0} \\
u_{j}^{*} u_{k}^{*}=\varepsilon_{j k l} u_{l} & u_{j}^{*} u_{k}=-\delta_{j k} u_{0}^{*} .
\end{array}
$$

Now $\partial \in L$ implies $\partial\left(u_{0}\right)=0=\partial\left(u_{0}^{*}\right)$. Moreover if $X$ denotes the span of the $u$ 's and $Y$ the span of the $u^{*}$ 's, then $X=\left\{x \in O_{c} \mid u_{0} x=\right.$ $\left.x=x u_{0}^{*}\right\}$ and $Y=\left\{y \in O_{c} \mid u_{0}^{*} y=y=y u_{0}\right\}$. It is easy to see from these characterizations that $X$ and $Y$ are $L_{c}$ invariant. From applying $\partial$ to the relation $u_{j} u_{k}^{*}=-\delta_{j k} u_{0}$, it follows that for each $\partial \in L_{c}$ the matrix of $\partial$ on $Y$ relative to the $u_{j}^{*}$ is minus the transpose of the matrix of $\partial$ on $X$ relative to the $u_{j}$. In addition the trace of $\partial$ on $X$ and on $Y$ must be 0 . These are the only restrictions on the elements of $L_{c}$. Thus $L_{c} \cong \operatorname{sl}(3)$, and $X$ is the module which we have been denoting by 3 (it is $3 \times 1$ matrices on which $\operatorname{sl}(3)$ acts by matrix multiplication), and $Y$ is $\overline{3}$ (it is $1 \times 3$ matrices on 
which the action of $\operatorname{sl}(3)$ is right multiplication by minus the matrix). From these observations it follows that if $Z$ denotes the span of $e_{1}, \cdots, e_{6}$ in $O$, then $L$ leaves $Z$ invariant, while our previous remarks show that $Z$ must be an irreducible module for $s u(3)$.

Let us consider the $L$-module homomorphisms of $Z \otimes Z$ into $Z$. Since $Z_{c}=X+Y$, and since $3 \otimes 3=6+\overline{3}, \overline{3} \otimes \overline{3}=\overline{6}+3$ and $3 \otimes \overline{3}=$ $8+1$ we see $\operatorname{dim}_{c} \operatorname{Hom}_{L_{C}}((X+Y) \otimes(X+Y), X+Y)=2$. It is spanned by the homomorphisms $\varphi_{1}, \varphi_{2}$ where $\varphi_{1}\left(u_{j} \otimes u_{k}\right)=\varepsilon_{j k l} u_{l}^{*}$, $\varphi_{2}\left(u_{j}^{*} \otimes u_{k}^{*}\right)=\varepsilon_{j k l} u_{l}$, and $\varphi_{1}$ and $\varphi_{2}$ are 0 on all products of basis elements not of the specified type.

Given $\varphi \in \operatorname{Hom}_{L}(Z \otimes Z, Z)$, then $\varphi$ lifts to an $L_{c}$-homomorphism of $(X+Y) \otimes(X+Y)$ into $X+Y$, and so $\varphi=a \varphi_{1}+b \varphi_{2}$ where $a, b \in C . \quad$ Therefore $\varphi\left(\left(u_{j}+u_{j}^{*}\right) \otimes\left(u_{k}+u_{k}^{*}\right)\right)=\varepsilon_{j k l}\left(a u_{l}^{*}+b u_{l}\right) . \quad$ But since $u_{j}+u_{j}^{*}$ and $u_{k}+u_{k}^{*}$ lie in $Z$, so does $a u_{l}^{*}+b u_{l}$, and $a u_{l}^{*}+$ $b u_{l}=\alpha\left(u_{l}+u_{l}^{*}\right)+\beta i\left(u_{l}^{*}-u_{l}\right)$ where $\alpha, \beta \in \boldsymbol{R}$. Thus $a=\alpha+\beta i$, $b=\alpha-\beta i$ and $b=\bar{a}$. It follows that

$$
\begin{aligned}
& \varphi\left(\left(u_{j}+u_{j}^{*}\right) \otimes\left(u_{k}+u_{k}^{*}\right)\right)=\varepsilon_{j k l}\left\{\alpha\left(u_{l}+u_{l}^{*}\right)+\beta i\left(u_{l}^{*}-u_{l}\right)\right\} \\
& \varphi\left(\left(u_{j}+u_{j}^{*}\right) \otimes i\left(u_{k}^{*}-u_{k}\right)\right)=\varepsilon_{j k l}\left\{\beta\left(u_{l}+u_{l}^{*}\right)-\alpha i\left(u_{l}^{*}-u_{l}\right)\right\} \\
& \varphi\left(i\left(u_{j}^{*}-u_{j}\right) \otimes\left(u_{k}+u_{k}^{*}\right)\right)=\varepsilon_{j k l}\left\{\beta\left(u_{l}+u_{l}^{*}\right)-\alpha i\left(u_{l}^{*}-u_{l}\right)\right\} \\
& \varphi\left(i\left(u_{j}^{*}-u_{j}\right) \otimes i\left(u_{k}^{*}-u_{k}\right)\right)=\varepsilon_{j k l}\left\{-\alpha\left(u_{l}+u_{l}^{*}\right)-\beta i\left(u_{l}^{*}-u_{l}\right)\right\} .
\end{aligned}
$$

These equations determine the effect of $\varphi$ on the $e_{r} \otimes e_{s}$ basis of $Z \otimes Z$.

Since the modules involved are unique up to isomorphism, the general case of an irreducible 6-dimensional su(3)-module $Z$ which becomes $3+\overline{3}$ upon complexification is no different from the behavior just observed. There is a basis $e_{1}, \cdots, e_{6}$ of $Z$ such that any $s u(3)-$ module homomorphism $\phi$ is given as above for some $\alpha, \beta \in \boldsymbol{R}$. If $Z$ is a summand in an algebra $A$ which admits $s u(3)$ as derivations, then these homomorphisms determine the possible products from $Z \times Z$ to $Z$, and since the homomorphisms are all skew-symmetric, the products will be anticommutative.

Thus we may assume that the products from $Z \times Z$ to $Z$ are given by (4.3) for some $\alpha, \beta \in \boldsymbol{R}$.

\begin{tabular}{ccccccc}
\multicolumn{1}{c}{$e_{1}$} & $e_{2}$ & $e_{3}$ & $e_{4}$ & $e_{5}$ & $e_{6}$ \\
\cline { 2 - 7 }$e_{1}$ & - & $\alpha e_{4}+\beta e_{5}$ & - & $-\alpha e_{2}-\beta e_{6}$ & $-\beta e_{2}+\alpha e_{6}$ & $\beta e_{4}-\alpha e_{5}$ \\
$e_{2}$ & $-\alpha e_{4}-\beta e_{5}$ & - & $-\beta e_{4}+\alpha e_{5}$ & $\alpha e_{1}+\beta e_{3}$ & $\beta e_{1}-\alpha e_{3}$ & - \\
$e_{3}$ & - & $\beta e_{4}-\alpha e_{5}$ & - & $-\beta e_{2}+\alpha e_{6}$ & $\alpha e_{2}+\beta e_{6}$ & $-\alpha e_{4}-\beta e_{5}$ \\
$e_{4}$ & $\alpha e_{2}+\beta e_{6}$ & $-\alpha e_{1}-\beta e_{3}$ & $\beta e_{2}-\alpha e_{6}$ & - & - & $-\beta e_{1}+\alpha e_{3}$ \\
$e_{5}$ & $\beta e_{2}-\alpha e_{6}$ & $-\beta e_{1}+\alpha e_{3}$ & $-\alpha e_{2}-\beta e_{6}$ & - & - & $\alpha e_{1}+\beta e_{3}$ \\
$e_{6}$ & $-\beta e_{4}+\alpha e_{5}$ & - & $\alpha e_{4}+\beta e_{5}$ & $\beta e_{1}-\alpha e_{3}$ & $-\alpha e_{1}-\beta e_{3}$ & - \\
\hline
\end{tabular}





and $u_{j}^{*} u_{k}^{*}=\varepsilon_{j k l} \bar{a} u_{l}$ where $a=\alpha+\beta i$. Let us suppose $v_{j}=$ $a^{-2 / 3} \bar{a}^{1 / 3} u_{j}$ and $v_{j}^{*}=a^{-1 / 3} \bar{a}^{2 / 3} u_{j}^{*}$ so that $v_{j} v_{k}=\varepsilon_{j k l} v_{l}^{*}$ and $v_{j}^{*} v_{k}^{*}=\varepsilon_{j k l} v_{l}$. Now let $z_{1}=v_{1}+v_{1}^{*}, z_{2}=v_{2}+v_{2}^{*}, z_{4}=v_{3}+v_{3}^{*}, z_{3}=i\left(v_{1}^{*}-v_{1}\right), z_{6}=$ $i\left(v_{2}^{*}-v_{2}\right), z_{5}=i\left(v_{3}^{*}-v_{3}\right)$. Then the multiplication table for the $z$ 's is the same as (4.3) when $\alpha=1$ and $\beta=0$. Note $z_{1}=\gamma e_{1}+\zeta e_{3}, z_{2}=$ $\gamma e_{2}+\zeta e_{6}, z_{4}=\gamma e_{4}+\zeta e_{5}, z_{3}=-\zeta e_{1}+\gamma e_{3}, z_{6}=-\zeta e_{2}+\gamma e_{6}, z_{5}=-\zeta e_{4}+\gamma e_{5}$ where $\gamma=1 / 2\left(a^{-2 / 3} \bar{a}^{-1 / 3}+\bar{a}^{-2 / 3} a^{-1 / 3}\right)$ and $\zeta=(1 / 2) i\left(a^{-2 / 3} \bar{a}^{-1 / 3}-\bar{a}^{-2 / 3} a^{-1 / 3}\right)$. Since $\bar{\gamma}=\gamma$ and $\bar{\zeta}=\zeta$, the $z$ 's lie in $Z$, and they are the desired basis.

To calculate further entries in the (4.2) table let us recall that $3 \otimes \overline{3}=8+1$. (This resolution can be concretely realized by the matrix multiplication of a $3 \times 1$ matrix with a $1 \times 3$ matrix followed by projection onto $\operatorname{sl}(3)$ and $C \cdot I)$. Thus, $v_{j} \otimes v_{k}^{*} \rightarrow \delta_{j k} w$ is an $\operatorname{sl}(3)$-module homomorphism of $3 \otimes \overline{3}$ onto the 1-dimensional module spanned by $w$, and any other one is just a complex multiple of this homomorphism. From this it follows that any $s u(3)$-module homomorphism $\psi_{1}: Z \times Z \rightarrow \boldsymbol{R} w$ when lifted to $(3+\overline{3}) \otimes(3+\overline{3}) \rightarrow$ $C w$ is given by $\psi_{1}\left(v_{j} \otimes v_{k}^{*}\right)=c \delta_{j k} w, \psi_{1}\left(v_{k}^{*} \otimes v_{j}\right)=d \delta_{j k} w$ for $c, d \in C$ and the condition $\psi_{1}(Z \otimes Z) \subseteq \boldsymbol{R} w$ forces $d=\bar{c}$. Thus if $c=\alpha_{1}+\beta_{1} i$

$$
\begin{aligned}
& \psi_{1}\left(\left(v_{j}+v_{j}^{*}\right) \otimes\left(v_{k}+v_{k}^{*}\right)\right)=2 \delta_{j k} \alpha_{1} w \\
& \psi_{1}\left(\left(v_{j}+v_{j}^{*}\right) \otimes i\left(v_{k}^{*}-v_{k}\right)\right)=2 \delta_{j k} \beta_{1} w \\
& \psi_{1}\left(i\left(v_{j}^{*}-v_{j}\right) \otimes\left(v_{k}^{*}+v_{k}\right)\right)=-2 \delta_{j k} \beta_{1} w \\
& \psi_{1}\left(i\left(v_{j}^{*}-v_{j}\right) \otimes i\left(v_{k}^{*}-v_{k}\right)\right)=2 \delta_{j k} \alpha_{1} w .
\end{aligned}
$$

Similarly if $R x$ is the other 1-dimensional summand any homomorphism $\psi_{2}$ is prescribed by scalars $\alpha_{2}, \beta_{2}$. Thus any product of $Z \times Z$ into the two 1-dimensional summands is determined by four scalars $\alpha_{1}, \beta_{1}, \alpha_{2}, \beta_{2} \in \boldsymbol{R}$. This number can be reduced by making the change of basis $u=-2 \alpha_{1} w-2 \alpha_{2} x, v=2 \beta_{1} w+2 \beta_{2} x$. For then $z_{j}^{2}=-u$ for all $j$ and $z_{1} z_{3}=z_{2} z_{6}=z_{4} z_{5}=v=-z_{3} z_{1}=-z_{6} z_{2}=-z_{5} z_{4}$ as in the table. The elements $u, v$ will seem less mysterious if one keeps the octonion example in mind, for there $v$ corresponds to $e_{7}$ and $u$ to the identity element.

The relations $1 \otimes 3=3,1 \otimes \overline{3}=\overline{3}$ similarly imply the existence of scalars $\sigma_{1}, \sigma_{2} \in R$ such that

$$
\begin{aligned}
& u\left(v_{j}+v_{j}^{*}\right)=\sigma_{1}\left(v_{j}+v_{j}^{*}\right)+\sigma_{2} i\left(v_{j}^{*}-v_{j}\right) \\
& u i\left(v_{j}^{*}-v_{j}\right)=-\sigma_{2}\left(v_{j}+v_{j}^{*}\right)+\sigma_{1} i\left(v_{j}^{*}-v_{j}\right) .
\end{aligned}
$$

In this fashion one obtains the entries in the table involving the $\sigma$ 's and $\tau$ 's. Since $\boldsymbol{R} u+\boldsymbol{R} v$ is a subalgebra, the products $u^{2}, u v$, $v u, v^{2}$ are of the form indicated by (4.2), and the determination of 
the table is complete. This table has been constructed by using $s u(3)$-modules and $s u(3)$-module homomorphisms at each stage, so that any algebra having (4.2) as its table for $\sigma^{\prime}$ 's, $\tau$ 's, $\eta^{\prime}$ 's, $\theta$ 's in $\boldsymbol{R}$ will admit $s u(3)$ as derivations.

If $A$ is a division algebra with multiplication given by (4.2) and if $\operatorname{Der} A$ is larger than $s u(3)$, then $\operatorname{Der} A$ is a compact $G_{2}$, since this is the only Lie algebra in our classification of derivation algebras of real division algebras which can properly contain $s u(3)$. The criterion for when Der $A$ is a compact $G_{2}$ is given in

THEOREM 4.4. If $A$ is a real division algebra with multiplication given by (4.2), then $\operatorname{Der} A$ is a compact form of $G_{2}$ if and only if the following relations hold:

$$
\begin{aligned}
& \eta_{2}=0=\eta_{3}, \theta_{1}=0=\theta_{4}, \theta_{2}=\sigma_{1}, \theta_{3}=\tau_{1}, \\
& \eta_{4}=-1=\tau_{4}, \sigma_{2}=0=\tau_{2}, \sigma_{3}=0=\tau_{3}, \sigma_{4}=1 .
\end{aligned}
$$

Otherwise Der $A=s u(3)$.

Proof. If the relations (4.5) hold, then it is immediate that $A$ is isomorphic to the algebra defined by (2.1) with $\zeta=\eta_{1}^{-1} \tau_{1}, \eta=\eta_{1}^{-1} \sigma_{1}$, and $\beta=\eta_{1}$ under the correspondence $u \leftrightarrow \eta_{1}^{-1} u, e_{7} \leftrightarrow v$, and $e_{j} \leftrightarrow z_{j}$ for $j=1, \cdots, 6$. Hence $\operatorname{Der} A$ is a compact $G_{2}$ in this case.

Conversely suppose $\operatorname{Der} A$ is a compact form of $G_{2}$. Then $A$ decomposes relative to $\operatorname{Der} A$ into a 1 -dimensional module $U$ and a 7 -dimensional irreducible module $V$ as in $\S 2$. Since $Z$ is the image of $A$ under $s u(3) \leqq$ Der $A, Z$ must be contained in $V$, the image of $A$ under Der $A$. Every element of $V$ is known to square to an element in $U$ (see Table 2.1), but every element of $Z$ squares to an multiple of $u$. Thus $U$ is the span of $u$, and $V$ is the span of the $z$ 's and $v+\lambda u$ for some $\lambda \in \boldsymbol{R}$. It follows that right or left multiplication by $u$ on $V$, and also on $U$, must be a scalar multiple of the identity transformation, and this implies that $\sigma_{2}=0_{1}=\tau_{2}, \theta_{1}=$ $0, \theta_{2}=\sigma_{1}$, and $\theta_{3}=\tau_{1}$. Since $A$ is a division algebra, left or right multiplication by a nonzero linear combination of $u$ and $v$ on $z_{1}$ must be nonzero, and this forces $\sigma_{4} \neq 0$ and $\tau_{4} \neq 0$.

We deduce further relations by recalling that products from $V \times V \rightarrow V$ are the same as in the octonions. Thus, they share the property that if $x, y$ and $y x$ are in $V$, then $(y x) x \in \boldsymbol{R} y$ and $y(y x) \in \boldsymbol{R} x$, since these properties follow from the alternativity of the octonions. (See for example, Schafer [9].) Such elements are $v+\lambda u, z_{1}$ and $(v+\lambda u) z_{1}=\left(\sigma_{3}+\lambda \sigma_{1}\right) z_{1}+\sigma_{4} z_{3}$, so that

$$
\left((v+\lambda u) z_{1}\right) z_{1}=-\left(\sigma_{3}+\lambda \sigma_{1}\right) u-\sigma_{4} v \in \boldsymbol{R}(v+\lambda u) .
$$




$$
\begin{gathered}
(v+\lambda u)\left((v+\lambda u) z_{1}\right)=\left(\left(\sigma_{3}+\lambda \sigma_{1}\right)\left(\sigma_{3}+\lambda \sigma_{1}\right)-\sigma_{4}^{2}\right) z_{1} \\
+2 \sigma_{4}\left(\sigma_{3}+\lambda \sigma_{1}\right) z_{4} \in R z_{1} .
\end{gathered}
$$

Since $\sigma_{4} \neq 0$, equation (4.7) implies that $\sigma_{3}+\lambda \sigma_{1}=0$, and this together with (4.6) says $-\sigma_{4} v \in \boldsymbol{R}(v+\lambda u)$. Hence $\lambda=0, \sigma_{3}=0$, and an analogous argument with $z_{1}\left(z_{1} v\right)$ determines that $\tau_{3}=0$. Further consequences of the result that $v \in V$ are $\eta_{2}=0=\eta_{3}$ and $\theta_{4}=0$, because $x^{2} \in U$ for each $x \in V$.

An additional property of $V$ inherited from the octonions is that if $y \in V$ and if for some $x \in V, x y \in V$ and $(x y) y=-\rho x$, for $\rho \in \boldsymbol{R}$, then for any $w \in V$ with $w y \in V,(w y) y=-\rho w$. Therefore, $\left(z_{2} z_{1}\right) z_{1}=-z_{2}$ and $\left(v z_{1}\right) z_{1}=-\sigma_{4} v$ imply $\sigma_{4}=1$, while by symmetry $z_{1}\left(z_{1} z_{2}\right)=-z_{2}$ and $z_{1}\left(z_{1} v\right)=\tau_{4} v$ give $\tau_{4}=-1$.

All that is left to be shown is that $\eta_{4}=-1$. However if $x, y$, and $x y$ are in $V$, and $(x y) y=-x$ and $x(x y)=-y$ then $x^{2}=y^{2}$, since the corresponding result holds for the octonions. But then $\left(v z_{1}\right) z_{1}=$ $-v$ and $v\left(v z_{1}\right)=-z_{1}$ imply $-u=z_{1}^{2}=v^{2}=\eta_{4} u$. From this we deduce that $\eta_{4}=-1$, so that all the conditions in (4.5) do indeed hold when Der $A=$ compact $G_{2}$.

The question of when a real algebra with multiplication given by (4.2) is a division algebra is formidable because of the large number of scalars in the multiplication table. However, we can exhibit division algebras of this type which have $s u(3)$ as their full derivation algebra. The easiest example is obtained by taking the values of the constants prescribed in (4.5) with the sole exception that $\eta_{4}$ is some negative number besides -1 . This algebra was shown to be a division algebra in ([1], Theorem 20), and it has $s u(3)$ as its derivation algebra according to Theorem 4.4.

5. The case $\operatorname{Der} A=s u(2) \oplus s u(2)$. An irreducible $s l(2) \oplus s l(2)$ module over $C$ is just the tensor product of two irreducible $s l(2)$ modules (one for each summand of $s l(2) \oplus \operatorname{sl}(2)$ ). If $V_{1}$ is an irreducible module for the first copy of $\operatorname{sl}(2)$, and $V_{2}$ for the second copy, and if $\operatorname{dim} V_{1}=m$ and $\operatorname{dim} V_{2}=n$, then $V_{1} \otimes V_{2}$ is an irreducible module for $s l(2) \oplus \operatorname{sl}(2)$ of dimension $m n$, and we denote this module by $m \widetilde{\otimes} n$.

Suppose now $s u(2) \oplus s u(2) \subseteq \operatorname{Der} A$ where $A$ is a real division algebra, and for convenience write $S_{1}$ and $S_{2}$ for the two copies of $s u(2)$. As we explained in $\S 1$, we have the $S_{1}$-module decomposition $A=A_{0} \oplus A_{*}$ where $A_{0}$ is the space of elements annihilated by $S_{1}$ and $A_{*}$ is the image of $A$ under $S_{1}$. Since $S_{1}$ and $S_{2}$ commute, it is easy to see that $A_{0}$ and $A_{*}$ are invariant under $S_{2}$. The $S_{2}$-action on $A_{0}$ and $A_{*}$ affords the decompositions, $A_{0}=A_{00} \oplus A_{0 *}$ and $A_{*}=$ $A_{* 0} \oplus A_{* *}$. Thus, relative to $S_{1} \oplus S_{2}$ 


$$
A=A_{00} \oplus A_{0 *} \oplus A_{* 0} \oplus A_{* *} .
$$

Since the smallest nontrivial $s u(2)$-module has dimension 3 , we see $A_{0 *}=0$ or $\operatorname{dim} A_{0 *} \geqq 3$, and the same is true of $A_{* 0}$. Because $\left(A_{* *}\right)_{c}$ is just the sum of all irreducible $s l(2) \oplus s l(2)$-modules not annihilated by either summand, it follows that either $A_{* *}=0$ or else $\operatorname{dim} A_{* *} \geqq 4$. We consider the various possibilities.

Of course, not all $A_{* 0}, A_{0 *}, A_{* *}$ can be zero, since $S_{1} \oplus S_{2}$ acts nontrivially on $A$. If $A_{0 *} \neq 0 \neq A_{* 0}$, then a simple dimension count shows $A_{* *}=0$. Since $(m \widetilde{\otimes} 1) \otimes(1 \widetilde{\otimes} n)=m \widetilde{\otimes} n$ for $\operatorname{sl}(2) \oplus \operatorname{sl}(2)$ modules, $A_{* 0} A_{0 *} \subseteq\left(A_{* 0}\right)_{c}\left(A_{0 *}\right)_{c} \leqq\left(A_{* *}\right)_{c}=0$. This contradiction enables us to conclude either $A_{* 0}=0$ or $A_{0 *}=0$. Without loss of generality we suppose that $A_{0 *}=0$, and hence $A=A_{00} \oplus A_{* 0} \oplus A_{* *}$. In this decomposition $A_{* *} \neq 0$, since otherwise $S_{2}$ would act trivially on $A$. Now $\left(A_{* *}\right)_{c}$ is comprised of a sum of modules of the following types: $2 \widetilde{\otimes} 2,2 \widetilde{\otimes} 3,3 \widetilde{\otimes} 2,2 \widetilde{\otimes} 4,4 \widetilde{\otimes} 2$. In any event, $\left(A_{* *}\right)_{c}$ is the direct sum of copies of modules of dimension 2 when it is decomposed relative to one of the copies of $\operatorname{sl}(2)$. Since $2 \otimes 2=3+1$ for $s l(2)$-modules it must be that $\left(A_{* *}\right)_{c}^{2} \subseteq\left(A_{00}\right)_{c}+\left(A_{* 0}\right)_{c}$, and hence $A_{* *}^{2} \subseteq A_{00}+A_{* 0}$. For any $x \neq 0$ in $A_{* *}, x A_{* *} \subseteq A_{00}+A_{* 0}$, and because left multiplication by $x$ is nonsingular, $\operatorname{dim} A_{* *} \leqq \operatorname{dim}\left(A_{00}+A_{* 0}\right)$. Thus, there is only one possibility for $\left(A_{* *}\right)_{c}$, namely $\left(A_{* *}\right)_{c}=2 \widetilde{\otimes} 2$.

If $\operatorname{dim} A_{00}=0$, then $\operatorname{dim} A_{* 0}=\operatorname{dim} A_{* *}=4$ and $\left(A_{c}\right)_{* 0}=2+2$ relative to $\left(S_{1}\right)_{c}=s l(2)$. But then $A_{C}$ is just the sum of 2-dimensional modules for $\left(S_{1}\right)_{c}$, and as above $2 \otimes 2=3+1$ shows that all products are zero. Thus, it is impossible for $A_{00}$ to be zero.

Consider now the possibility $A_{* 0}=0$. In this instance $\operatorname{dim} A_{00}=$ $\operatorname{dim} A_{* *}=4$, and every derivation of $A$ in $S_{1} \oplus S_{2}$ has rank $\leqq 4$. If this is the case, then any space of commuting derivations has dimension not more than one according to ([1], Corollary 16). However, $S_{1} \oplus S_{2}$ has a 2-dimensional space of commuting derivations, so we arrive at a contradiction. Therefore $A_{* 0} \neq 0$, and since $A_{00} \neq 0$ and $\operatorname{dim} A_{* *}=4$, it must be that $\operatorname{dim} A_{* 0}=3$. This is the first part of the principal result of this section which we are now ready to state and prove.

THEOREM 5.1. Let $A$ be a real division algebra such that $s u(2) \oplus s u(2) \subseteq \operatorname{Der} A$. Then as an $s u(2) \oplus s u(2)-m o d u l e, A$ is a direct sum of a 1-dimensional module $U$ annihilated by both copies of su(2), a 3-dimensional module $X$ irreducible under one copy of su(2) and annihilated by the other, and a 4-dimensional module $Y$ irreducible under both copies of su(2). There exists a basis $u, x_{1}, x_{2}, x_{3}, y_{1}, y_{2}, y_{3}, y_{4}$ such that the multiplication in $A$ is given by (5.2) for some $\beta, \gamma, \delta, \varepsilon, \eta$, $\zeta, \theta, \rho, \sigma \in R$. Furthermore, Der $A$ is either su(2) $\oplus s u(2)$ or a compact 
$G_{2}$; the latter occurring exactly when the following relations hold: $\varepsilon=1=\eta, \beta \gamma=\delta, \zeta=\rho, \theta=\sigma, \gamma>0$, and $\beta \rho \sigma<0$.

\begin{tabular}{|c|c|c|c|c|c|c|c|c|}
\hline & $u$ & $x_{1}$ & $x_{2}$ & $x_{3}$ & $y_{1}$ & $y_{2}$ & $y_{3}$ & $y_{4}$ \\
\hline$u$ & $u$ & $\zeta x_{1}$ & $\zeta x_{2}$ & $\zeta x_{3}$ & $\rho y_{1}$ & $\rho y_{2}$ & $\rho y_{3}$ & $\rho y_{4}$ \\
\hline$x_{1}$ & $\theta x_{1}$ & $\beta u$ & $x_{3}$ & $-x_{2}$ & $\varepsilon y_{4}$ & $\varepsilon y_{3}$ & $-\varepsilon y_{2}$ & $-\varepsilon y_{1}$ \\
\hline$x_{2}$ & $\theta x_{2}$ & $-x_{3}$ & $\beta u$ & $x_{1}$ & $\varepsilon y_{2}$ & $-\varepsilon y_{1}$ & $\varepsilon y_{4}$ & $-\varepsilon y_{3}$ \\
\hline$x_{3}$ & $\theta x_{3}$ & $x_{2}$ & $-x_{1}$ & $\beta u$ & $-\varepsilon y_{3}$ & $\varepsilon y_{4}$ & $\varepsilon y_{1}$ & $-\varepsilon y_{2}$ \\
\hline$y_{1}$ & $\sigma y_{1}$ & $-\eta y_{4}$ & $-\eta y_{2}$ & $\eta y_{3}$ & $\delta u$ & $\gamma x_{2}$ & $-\gamma x_{3}$ & $\gamma x_{1}$ \\
\hline$y_{2}$ & $\sigma y_{2}$ & $-\eta y_{3}$ & $\eta y_{1}$ & $-\eta y_{4}$ & $-\gamma x_{2}$ & $\delta u$ & $\gamma x_{1}$ & $\gamma x_{3}$ \\
\hline$y_{3}$ & $\sigma y_{3}$ & $\eta y_{2}$ & $-\eta y_{4}$ & $\eta y_{1}$ & $\gamma x_{3}$ & $-\gamma x_{1}$ & $\delta u$ & $\gamma x_{2}$ \\
\hline$y_{4}$ & $\sigma y_{4}$ & $\eta y_{1}$ & $\eta y_{3}$ & $\eta y_{2}$ & $-\gamma x_{1}$ & $-\gamma x_{3}$ & $-\gamma x_{2}$ & $\delta u$ \\
\hline
\end{tabular}

Proof. Recall from $\S 1$ that $\partial_{1}=i h, \partial_{2}=e-f \partial_{3}=i(e+f)$ give a basis of $s u(2)$ such that $\left[\partial_{j}, \partial_{j+1}\right]=2 \partial_{j+2}$ where the indices are interpreted modulo 3 . Let $\partial_{1}, \partial_{2}, \partial_{3}$ denote such a basis for the copy of $s u(2)$ which acts irreducibly on $X$, and let $\partial_{1}^{\prime}, \partial_{2}^{\prime}, \partial_{3}^{\prime}$ be the corresponding basis for the other copy. Since the module $X$ is just the adjoint representation of $s u(2)$, there is a basis $x_{1}, x_{2}, x_{3}$ of $X$ such that the action of $s u(2) \oplus s u(2)$ on $X$ is given by

$$
\begin{array}{ll}
\partial_{j}\left(x_{j+1}\right)=2 x_{j+2} & \text { where the subscripts are read modulo } 3 \\
\partial_{j}^{\prime}\left(x_{k}\right)=0 & \text { for all } j \text { and } k .
\end{array}
$$

Now $Y_{c}=2 \widetilde{\otimes} 2$, or in the notation of $\S 1, \quad Y_{c}=V(1) \otimes V(1)$. Recall $V(1) \otimes V(1)$ has as basis $\left\{v_{j} \otimes v_{k}\right\}$ where $j, k= \pm 1$. Let

$$
\begin{aligned}
& y_{1}=v_{1} \otimes i v_{1}+i v_{1} \otimes v_{1}+v_{-1} \otimes i v_{-1}+i v_{-1} \oplus v_{-1} \\
& y_{2}=v_{-1} \otimes i v_{1}+i v_{-1} \otimes v_{1}-v_{1} \otimes i v_{-1}-i v_{1} \otimes v_{-1} \\
& y_{3}=i v_{-1} \otimes i v_{1}-v_{-1} \otimes v_{1}+i v_{1} \otimes i v_{-1}-v_{1} \otimes v_{-1} \\
& y_{4}=v_{1} \otimes v_{1}-i v_{1} \otimes i v_{1}-v_{-1} \otimes v_{-1}+i v_{-1} \otimes i v_{-1} .
\end{aligned}
$$

Then $y_{1}, y_{1}, y_{3}, y_{4}$ span an $s u(2) \oplus s u(2)$-module as the table below indicates.

\begin{tabular}{r|rrrr|}
\multicolumn{1}{c}{} & $y_{1}$ & $y_{2}$ & $y_{3}$ & $y_{4}$ \\
\hline$\partial_{1}$ & $-y_{4}$ & $-y_{3}$ & $y_{2}$ & $y_{1}$ \\
$\partial_{2}$ & $-y_{2}$ & $y_{1}$ & $-y_{4}$ & $y_{3}$ \\
$\partial_{3}$ & $y_{3}$ & $-y_{4}$ & $-y_{1}$ & $y_{2}$ \\
$\partial_{1}^{\prime}$ & $-y_{4}$ & $y_{3}$ & $-y_{2}$ & $y_{1}$ \\
$\partial_{2}^{\prime}$ & $y_{2}$ & $-y_{1}$ & $-y_{4}$ & $y_{3}$ \\
$\partial_{3}^{\prime}$ & $y_{3}$ & $y_{4}$ & $-y_{1}$ & $-y_{2}$ \\
\hline
\end{tabular}


Thus, $Y$ must be isomorphic to the span of the $y$ 's.

In order to deduce the products $X^{2}, U X$, and $X U$, we recall that for $\operatorname{sl}(2)$-modules $3 \otimes 3=5+3+1$, and $3 \otimes 1=3$ (see (1.6)). Thus, $X^{2} \subseteq X+U$, and $X U+U X \subseteq X$, and up to scalar multiple there is just one possible product in each case. The product from $X \times X$ to $X$ is just the Lie product on $s u(2)$, from $X \times X$ to $U$ the product is simply the inner product (as seen from the quaternions on which $s u(2)$ acts as derivations), and from $X \times U$ to $X$ or $U \times X$ to $X$ the product is just multiplication by a scalar. After replacing each $x_{i}$ by an appropriate scalar multiple of itself, we obtain the portion of the multiplication table (5.2) pertaining to products on $X+U$.

Now for products involving $Y$, we have $(3 \widetilde{\otimes} 1) \otimes(2 \widetilde{\otimes} 2)=$ $(3 \otimes 2) \widetilde{\otimes}(1 \otimes 2)=(4+2) \widetilde{\otimes} 2=4 \widetilde{\otimes} 2+2 \widetilde{\otimes} 2$. Thus, $X Y+Y X \subseteq Y$, $\operatorname{dim}_{c} \operatorname{Hom}_{s l(2) \oplus s l(2)}\left(X_{c} \otimes Y_{c}, Y_{c}\right)=1$, and consequently

$$
\operatorname{dim}_{R} \operatorname{Hom}_{s u(2) \oplus s u(2)}(X \otimes Y, Y) \leqq 1 .
$$

Moreover, $\quad(2 \widetilde{\otimes} 2) \otimes(2 \widetilde{\otimes} 2)=(3+1) \widetilde{\otimes}(3+1)$ demonstrates that $Y^{2} \subseteq X+U, \operatorname{dim}_{R} \operatorname{Hom}_{s u(2) \oplus s u(2)}(Y \otimes Y, X) \leqq 1$, and $\operatorname{dim}_{R} \operatorname{Hom}_{s u(2) \oplus s u(2)}(Y \otimes$ $Y, U) \leqq 1$. Finally $(1 \widetilde{\otimes} 1) \otimes(2 \widetilde{\otimes} 2)=2 \widetilde{\otimes} 2$ shows that constants $\sigma, \rho \in \boldsymbol{R}$ exist so that $u y_{j}=\rho y_{j}$ and $y_{j} u=\sigma y_{j}$ for all $j$.

In order to find the products $X Y, Y X$, and $Y^{2}$ we again turn to the octonions for guidance. It is known that the transformations

$$
D_{v, w}=-a d_{[v, w]}+3\left[L_{v}, R_{w}\right]
$$

are derivations of the octonions for any two elements $v, w$ in the octonion algebra, where $L_{v}(t)=v t, R_{w}(t)=t w$, and $a d_{[v, w]}(t)=[[v w] t]$. (See [7, page 2].) Using the fact that $\left[\partial, D_{v, w}\right]=D_{\partial(v), w}+D_{v, \partial(w)}$, one can verify readily that

$$
\begin{aligned}
& \partial_{1}=-\frac{1}{2} D_{e_{2}, e_{4}}, \partial_{2}=-\frac{1}{2} D_{e_{1}, e_{1}}, \partial_{3}=-\frac{1}{2} D_{e_{1}, e_{2}} \\
& \partial_{1}^{\prime}=\frac{1}{2}\left(D_{e_{3}, e_{7}}-D_{e_{5}, e_{0}}\right), \partial_{2}^{\prime}=\frac{1}{2}\left(D_{e_{6}, e_{7}}-D_{e_{3}, e_{5}}\right), \partial_{3}^{\prime}=\frac{1}{2}\left(D_{e_{6}, e_{3}}-D_{e_{5}, e_{7}}\right)
\end{aligned}
$$

span a $s u(2) \oplus s u(2)$ subalgebra of the derivation algebra of the octonions with multiplication as above. Moreover, if one makes the following identifications $u \leftrightarrow 1, x_{1} \leftrightarrow e_{1}, x_{2} \leftrightarrow e_{2}, x_{3} \leftrightarrow e_{4}, y_{1} \leftrightarrow e_{3}, y_{2} \leftrightarrow e_{5}$, $y_{3} \leftrightarrow e_{8}$, and $y_{4} \leftrightarrow e_{7}$, the action of $s u(2) \oplus s u(2)$ on the $x$ 's and $y$ 's is exactly that given by (5.3) and (5.4). Therefore, since there is at most one $s u(2) \oplus s u(2)$-homomorphism up to scalar multiple in each of the cases: $X \otimes Y \rightarrow Y, Y \otimes X \rightarrow Y, Y \otimes Y \rightarrow X$, and $Y \otimes Y \rightarrow U$, the homomorphism can be computed easily from the corresponding products in the octonions. This calculation gives the remaining entries in (5.2). 
If Der $A$ properly contains $s u(2) \oplus s u(2)$ for a division algebra $A$ with multiplication given by (5.2), then Der $A$ must be a compact $G_{2}$, since this is the only Lie algebra in our classification of derivation algebras of real division algebras which can properly contain $s u(2) \oplus s u(2)$. The proof of Theorem 5.1 will be complete if we can show that $\operatorname{Der} A$ is a compact $G_{2}$ if and only if

$$
\varepsilon=1=\eta, \beta \gamma=\delta, \zeta=\rho, \theta=\sigma, \gamma>0 \text {, and } \beta \rho \sigma<0 \text {. }
$$

If $A$ satisfies the relations (5.5), then (5.2) reduces to the multiplication given in (2.1) under the correspondence given by $x_{1} \leftrightarrow e_{1}$, $x_{2} \leftrightarrow e_{2}, x_{3} \leftrightarrow e_{4}, y_{1} \leftrightarrow \sqrt{\gamma} e_{3}, y_{2} \leftrightarrow \sqrt{\gamma} e_{5}, y_{3} \leftrightarrow \sqrt{\gamma} e_{6}, y_{4} \leftrightarrow \sqrt{\gamma} e_{7}$, and so Der $A=$ compact $G_{2}$ when the relations (5.5) hold.

Conversely, suppose that $\operatorname{Der} A$ is a compact $G_{2}$ for a certain choice of the constants in (5.2). Then $A$ is isomorphic to one of the algebras of the form (2.1), and this isomorphism $\varphi$ must take $X+Y$ onto $V=\left\langle e_{1}, e_{2}, \cdots, e_{7}\right\rangle$. Now $V$ inherits from the alternativity of the octonions the property that if $v_{1}, v_{2} \in V$ and if $v_{1} v_{2} \in V$ then $v_{1}\left(v_{1} v_{2}\right) \in\left\langle v_{2}\right\rangle$. The same property must also hold for $\varphi^{-1}(V)=X+Y$, so that using (5.2) we obtain

$$
\begin{aligned}
\left(x_{2}+y_{1}\right)\left(\left(x_{2}+y_{1}\right) x_{1}\right)= & \left(x_{2}+y_{1}\right)\left(-x_{3}-\eta y_{4}\right)=-(1+\gamma \eta) x_{1} \\
& +(\varepsilon \eta-\eta) y_{3} \in\left\langle x_{1}\right\rangle .
\end{aligned}
$$

Thus, $\varepsilon \eta-\eta=0$, and $\varepsilon=1$ because $\eta \neq 0$ in a division algebra. Since $V$ is anticommutative, we also have $\eta=\varepsilon$, and so $\eta=1$. Then,

$$
\begin{aligned}
\left(x_{2}+y_{1}\right)\left(\left(x_{2}+y_{1}\right) y_{2}\right)= & \left(x_{2}+y_{1}\right)\left(-y_{1}+\gamma x_{2}\right)=-(1+\gamma) y_{2} \\
& +(\beta \gamma-\delta) u \in\left\langle y_{2}\right\rangle,
\end{aligned}
$$

giving $\beta \gamma=\delta$. Since left multiplication by $u$ is just a multiple of the identity on $V$, it follows the $\zeta=\rho$, and similarly $\theta=\sigma$. If $\gamma<0$, then $\sqrt{-\gamma}$ is a real number and

$$
\begin{gathered}
\left.\left.\left(\sqrt{-\gamma} u+\rho y_{1}\right)\right) \sqrt{-\gamma} x_{3}-y_{3}\right)=-\gamma \rho x_{3}-\sqrt{-\gamma} \rho y_{3} \\
+\sqrt{-\gamma} \rho y_{3}+\gamma \rho x_{3}=0
\end{gathered}
$$

using (5.2). Hence, $\gamma>0$ when $A$ is a division algebra. Finally, for any $c \in \boldsymbol{R}$,

$$
\begin{aligned}
\left(\sigma u+c x_{1}\right)\left(\sigma u-c x_{1}\right) & =\rho \sigma u-\rho \sigma c x_{1}+\rho \sigma c x_{1}-\beta c^{2} u \\
& =\left(\rho \sigma-\beta c^{2}\right) u,
\end{aligned}
$$

using $\zeta=\rho$ and $\theta=\sigma$. If $\beta \rho \sigma>0$, we can set $c=\sqrt{\beta^{-1} \rho \sigma}$ in the last calculation and obtain zero divisors. Thus, $\beta \rho \sigma<0$ in a division algebra, and we have verified all the relations of (5.5). 
Although we shall not attempt to derive necessary and sufficient conditions on the constants for the algebra $A$ given by (5.2) to be a division algebra, we note that there do exist division algebras of this form with $\operatorname{Der} A=s u(2) \oplus s u(2)$. For example, if we choose $\varepsilon=1=\eta=\gamma, \beta<0, \beta \neq \delta<0, \zeta=\rho=1=\theta=\sigma$, then $A$ is isomorphic to the division algebra of ([1], Theorem 20) using the map $u \leftrightarrow u, x_{1} \leftrightarrow e_{1}, x_{2} \leftrightarrow e_{2}, x_{3} \leftrightarrow e_{4}, y_{1} \leftrightarrow e_{3}, y_{2} \leftrightarrow e_{5}, y_{3} \leftrightarrow e_{6}, y_{4} \leftrightarrow e_{7}$.

6. The case $\operatorname{Der} A=s u(2)$ and $\operatorname{Cer} A=s u(2)+N$. Suppose now $A$ is a real division algebra and that $s u(2) \subseteq \operatorname{Der} A$. Using the convention explained in $\S 1$ of denoting an irreducible $s u(2)$ module by its dimension, we can state

Proposition 6.1. If $A$ is a real division algebra such that $s u(2) \subseteq \operatorname{Der} A$, then the decomposition of $A$ into irreducible su(2)modules has one of the following forms: $1+3,1+7,3+5,1+1+$ $3+3,1+3+4,1+1+1+1+4$.

Proof. We suppose first that $A$ is a direct sum of odd-dimensional irreducible modules. At least one irreducible module of dimension $\geqq 3$ must be present, since $s u(2)$ cannot act trivially on all of $A$. Then the only possibility when $\operatorname{dim} A=4$ is $1+3$. For $\operatorname{dim} A=8$, we note that the elements of $A$ annihilated by all of $s u(2)$ form a subalgebra which has dimension $0,1,2$, or 4 . With this restriction on the number of 1's in the decomposition, it is immediate that the only possible decompositions are $1+7,3+5$, and $1+1+3+3$.

Suppose then that $A$ has an even-dimensional irreducible module. Since by (1.6) the product of even-dimensional irreducible modules in $A_{c}$ must lie in the sum of the odd-dimensional irreducible modules, the same is true in $A$. Thus $A$ must also have odd-dimensional irreducible modules. In fact, the dimension of the sum of the odddimensional irreducible modules must be the same as the dimension of the sum of the even-dimensional modules, since right multiplication by any nonzero element of an even-dimensional irreducible module will map each of these two spaces into the other. As the smallest even-dimensional irreducible $s u(2)$-module has dimension 4 , it follows that $\operatorname{dim} A=8$ and that $A$ is the sum of a single 4dimensional irreducible module and some odd-dimensional irreducible modules. The only possibilities are $1+3+4$ and $1+1+1+$ $1+4$.

We discuss in turn each of the cases that arise in Proposition 6.1 beginning with the case $1+3$. This case is very similar to the 
case when $\operatorname{Der} A=$ compact $G_{2}$, since we see that there is exactly one product from $3 \times 3$ to 3 and one from $3 \times 3$ to 1 . Then $A$ is just like the quaternions except that there are several constants in the table. Specifically the multiplication table for $A$ is given by

\begin{tabular}{c|cccc|}
\multicolumn{1}{c}{} & \multicolumn{1}{c}{$u$} & $e_{1}$ & $e_{2}$ & $e_{4}$ \\
\cline { 2 - 5 }$u$ & $u$ & $\eta e_{1}$ & $\eta e_{2}$ & $\eta e_{4}$ \\
$e_{1}$ & $\zeta e_{1}$ & $-\beta u$ & $e_{4}$ & $-e_{2}$ \\
$e_{2}$ & $\zeta e_{2}$ & $-e_{4}$ & $-\beta u$ & $e_{1}$ \\
$e_{4}$ & $\zeta e_{4}$ & $e_{2}$ & $-e_{1}$ & $-\beta u$ \\
\hline
\end{tabular}

where we have normalized $e_{1}, e_{2}, e_{4}$ to make the scalar involved in the product from $3 \times 3$ to 3 become 1 , and we have normalized $u$ so that $u^{2}=u$. Since this algebra is a subalgebra of the algebra given by (2.1), it is a division algebra if $\beta \eta \zeta>0$ by Theorem 2.2. Conversely, if the algebra given by $(6.2)$ is a division algebra, then the equation

$$
0=\left(a_{0} u+a_{1} e_{1}+a_{2} e_{2}+a_{4} e_{4}\right)\left(b_{0} u+b_{1} e_{1}+b_{2} e_{2}+b_{4} e_{4}\right)
$$

can hold only if either all the $a$ 's or all the $b$ 's are zero. An argument identical to the proof of Theorem 2.2 shows that this condition implies $\beta \eta \zeta>0$. We have proved

THEOREM 6.3. A 4-dimensional real algebra is a division algebra with su(2) as its derivation algebra if and only if $A$ has a basis $u, e_{1}, e_{2}, e_{4}$ with multiplication given by (6.2) for some real numbers $\beta, \eta, \zeta$ such that $\beta \eta \zeta>0$.

The best known algebra belonging to the class defined by (6.2) is of course the algebra of quaternions, which arises by taking $\beta=\eta=\zeta=1$. If we take $\beta=1$ and $\eta=-1=\zeta$, we obtain the pseudo-quaternions of Okubo [8].

We consider next the case when $A$ has the decomposition $1+7$. Here we can establish

THEOREM 6.4. If $A$ is a real division algebra with su(2)@Der $A$, and if $A$ breaks up as an su(2)-module into a sum of a 1-dimen. sional module and an irreducible 7-dimensional module, then Der $A$ is a compact $G_{2}$. Hence the structure of $A$ is described by Theorem 2.2.

Proof. Let $A$ be an algebra satisfying the hypotheses of Theorem 6.4, let $U$ be the 1-dimensional module, and let $E$ be the 
irreducible 7-dimensional module. Then $U \otimes U \cong U$, and so $U$ is a subalgebra spanned by an idempotent $u$. Also, $U \otimes E \cong E$, and right multiplication by $u$ acts on $E$ as a scalar multiple of the identity transformation. Similarly, left multiplication by $u$ acts on $E$ as a scalar multiple of the identity. By the Clebsch-Gordan formula, there is up to a scalar multiple exactly one homomorphism from $E \otimes E$ to $E$, and exactly one from $E \otimes E$ to $U$. If we can show that these are the same two homomorphisms which come out of the algebras defined by (2.1), we will have shown that the present algebra $A$ belongs to the class of algebras defined by (2.1). In order to demonstrate that these homomorphisms are the same, it is sufficient to exhibit an algebra which satisfies the hypotheses of Theorem 6.4 and which also has the form (2.1), since the modules involved are unique up to isomorphism. Thus, it suffices to establish that the octonions $O$ satisfy the hypotheses of Theorem 6.4.

Letting $O$ be spanned by $u, e_{1}, \cdots, e_{7}$ where multiplication is given by (2.1) with $\beta=\eta=\zeta=1$, we show that there exists a subalgebra of Der $O$ isomorphic to $s u(2)$ which acts irreducibly on $E=\left\langle e_{1}, \cdots, e_{7}\right\rangle$. As we noted in $\S 5$, the maps

$$
D_{i j}=-a d_{\left[e_{i}, e_{j}\right]}+3\left[L_{e_{i}}, \boldsymbol{R}_{e_{j}}\right]
$$

are known to be derivations of $\boldsymbol{O}$. Then the linear transformations

$$
\begin{aligned}
& \partial_{1}=\frac{1}{3} D_{2,6}-\frac{4}{3} D_{4,5} \\
& \partial_{2}=-\frac{1}{2} \sqrt{6} D_{3,7}+\frac{1}{6} \sqrt{10}\left(D_{1,2}-D_{6,3}\right) \\
& \partial_{3}=\frac{1}{2} \sqrt{6} D_{7,1}+\frac{1}{6} \sqrt{10}\left(D_{6,1}-D_{2,3}\right)
\end{aligned}
$$

are also derivations of $\boldsymbol{O}$, and one can verify that the action of the $\partial_{j}$ 's on $E$ is given by

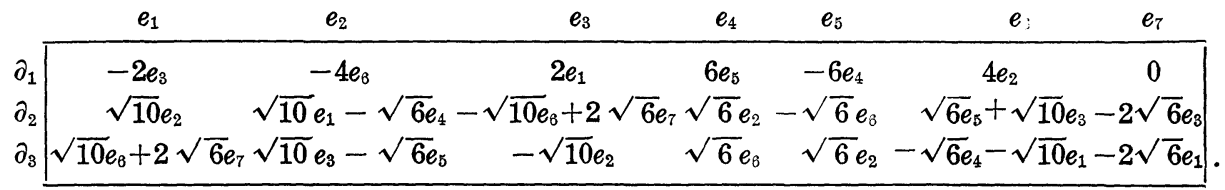

It is straightforward to check using this table that $\left[\partial_{j}, \partial_{j+1}\right]=2 \partial_{j+2}$ where the subscripts are interpreted modulo 3 . Thus, $\partial_{1}, \partial_{2}, \partial_{3}$ span a subalgebra of Der $O$ which is isomorphic to $s u(2)$.

It remains to show that $E$ is irreducible under this copy $S$ of $s u(2)$. We show first that each basis element $e_{j}$ generates all of $E$ under the action of $S$. Let $M\left(e_{j}\right)$ denote the $S$-submodule of $E$ 
generated by $e_{j}$. From the action of $\partial_{1}$, we see that $M\left(e_{1}\right)=M\left(e_{3}\right)$, $M\left(e_{2}\right)=M\left(e_{6}\right)$, and $M\left(e_{4}\right)=M\left(e_{5}\right)$. Since $\partial_{2}\left(e_{4}\right)=\sqrt{6} e_{2}$ and $\partial_{2}\left(e_{2}\right)=\sqrt{10} e_{1}-$ $\sqrt{6} e_{4}$, we have $e_{1}, \cdots, e_{6} \in M\left(e_{4}\right)$. Also, $\partial_{2}\left(e_{2}\right)=\sqrt{10} e_{1}-\sqrt{6} e_{4}$ and $\partial_{3}\left(e_{6}\right)=-\sqrt{6} e_{4}-\sqrt{10} e_{1}$ imply that $e_{1}, \cdots, e_{6} \in M\left(e_{2}\right)=M\left(e_{6}\right)$. Similarly, we obtain $e_{1}, \cdots, e_{6} \in M\left(e_{1}\right)=M\left(e_{3}\right)$. Since any submodule containing $e_{1}, \cdots, e_{6}$ contains $e_{7}$ using $\partial_{3}\left(e_{1}\right)=\sqrt{10} e_{6}+2 \sqrt{6} e_{7}$, we see that $M\left(e_{1}\right)=$ $M\left(e_{2}\right)=\cdots=M\left(e_{8}\right)=E$. Then $M\left(e_{7}\right)=E$ also, because $e_{1} \in M\left(e_{7}\right)$ follows from $\partial_{3}\left(e_{7}\right)=2 \sqrt{6} e_{1}$.

If $E$ is not an irreducible $S$-module, there exists an element which generates a nonzero proper submodule, and among all such elements we pick one, $w=\lambda_{1} e_{1}+\cdots+\lambda_{7} e_{7}$, of shortest length (i.e., with as many $\lambda$ 's zero as possible). It is easy to see that the element

$$
\begin{array}{r}
w_{1}=\alpha^{2} w+\partial_{1}^{2} w=\left(\alpha^{2}-4\right) \lambda_{1} e_{1}+\left(\alpha^{2}-16\right) \lambda_{2} e_{2}+\left(\alpha^{2}-4\right) \lambda_{3} e_{3} \\
+\left(\alpha^{2}-36\right) \lambda_{4} e_{4}+\left(\alpha^{2}-36\right) \lambda_{5} e_{5}+\left(\alpha^{2}-16\right) \lambda_{6} e_{6}+\alpha^{2} \lambda_{7} e_{7}
\end{array}
$$

will have shorter length than $w$ for some $\alpha \in\{0,2,4,6\}$ and that $M\left(w_{1}\right) \leqq M(w) \neq E$. Since $w$ has the shortest length among all nonzero elements, we obtain $w_{1}=0$ for some $\alpha$, which implies that $w$ has one of the forms

$$
\lambda_{1} e_{1}+\lambda_{3} e_{3}, \lambda_{2} e_{2}+\lambda_{8} e_{6}, \lambda_{4} e_{4}+\lambda_{5} e_{5}, \lambda_{7} e_{7} .
$$

The case when $w=\lambda_{7} e_{7}$ has already been eliminated. If $w=\lambda_{1} e_{1}+$ $\lambda_{3} e_{3}$, then $2 \lambda_{1} w+\lambda_{3} \partial_{1} w=\left(2 \lambda_{1}^{2}+2 \lambda_{3}^{2}\right) e_{1}$, showing that $e_{1}$ is in the submodule generated by $w$. But we have shown that $e_{1}$ generates all of $E$, so $w$ could not be of the form $\lambda_{1} e_{1}+\lambda_{3} e_{3}$. An identical argument rules out the cases when $w=\lambda_{2} e_{2}+\lambda_{6} e_{6}$ and $w=\lambda_{4} e_{4}+\lambda_{5} e_{5}$. Thus $E$ is an irreducible $S$-module.

We turn now to the case when $A$ is a direct sum of an irreducible 3-dimensional $s u(2)$-module and an irreducible 5-dimensional $s u(2)$-module. Since each of $3 \times 3,3 \times 5,5 \times 3,5 \times 5$ has one multiplication into each of 3 and 5 , there will be eight constants in the multiplication table of $A$. One can construct $A$ by thinking of $A$ as the $3 \times 3$ skew-Hermitian complex matrices of trace zero, where both $s u(2)$ and the 3-dimensional submodule of $A$ are identified with the subspace of matrices which are skew (as well as skewHermitian), and where the 5-dimensional module is those matrices which are symmetric (and skew-Hermitian). The action of $s u(2)$ on the two modules is the Lie product, and the different multiplications between the two modules in $A$ are obtained by resolving into the 3 and 5-components the two products on this set of matrices given in (3.1). 
As is obvious from the construction of $A$, the algebras occurring here include the class of algebras studied in $\S 3$. On the other hand, when $A$ has no 1-dimensional submodule for $s u(2)$, it cannot have a 1-dimensional submodule for all of $\operatorname{Der} A$, which rules out the cases that $\operatorname{Der} A$ is either a compact $G_{2}$ or $s u(2) \oplus s u(2)$, and the case when $\operatorname{Der} A=s u(3)$ and $A$ is not an irreducible $s u(3)$ module. We have established most of

THEOREM 6.5. If $A$ is a real division algebra with $s u(2) \subseteq$ Der $A$, and if $A$ is the sum of an irreducible 3-dimensional su(2)-module and an irreducible 5-dimensional su(2)-module, then either $\operatorname{Der} A=s u(2)$, or else $\operatorname{Der} A=s u(3)$ and $A$ is an irreducible su(3)module.

Proof. In view of our classification of the derivation algebras of division algebras and of the remarks in the paragraph before the statement of the theorem, it is only necessary to rule out the case that $\operatorname{Der} A=s u(2) \oplus N$ where $N$ is a 1-dimensional Lie algebra. Employing the representation of $A$ explained above, we let $\partial_{1}, \partial_{2}, \partial_{3}$ be the basis of $s u(2)$ and $x_{1}, x_{2}, x_{3}$ the basis for the 3 -dimensional module $X$ defined by

$$
\partial_{1}=e_{12}-e_{21}=x_{1}, \partial_{2}=e_{23}-e_{32}=x_{2}, \partial_{3}=e_{13}-e_{31}=x_{3},
$$

where the $e_{i j}$ 's are $3 \times 3$ matrix units. We let

$$
\begin{gathered}
y_{1}=i\left(e_{12}+e_{21}\right), y_{2}=i\left(e_{23}+e_{32}\right), y_{3}=i\left(e_{13}+e_{31}\right), \\
y_{4}=i\left(e_{11}-e_{22}\right), y_{5}=i\left(e_{22}-e_{33}\right)
\end{gathered}
$$

be the basis of the 5-dimensional module $Y$.

If $\operatorname{Der} A=s u(2) \oplus N$, then there exists a nonzero derivation $\partial$ commuting with $\partial_{1}, \partial_{2}, \partial_{3}$. By ([1], Lemma 15), the rank of any derivation on an 8-dimensional real division algebra is 0,4 , or 6 . But $\partial(A)$ is an $s u(2)$-submodule and so must have dimension $0,3,5$, or 8. Hence, $\partial(A)=0$, and $\partial=0$. This rules out the case $\operatorname{Der} A=$ $s u(2)+N$ here.

REMARK. The question of whether real division algebras satisfying the hypotheses of Theorem 6.5 and having Der $A=s u(2)$ actually exist has not been settled, to the best of our knowledge.

Consider next the case when the decomposition of $A$ as $s u(2)-$ modules is $1+1+3+3$. By determining all possible homomorphisms from the tensor product of two summands into a third summand, one can obtain a general multiplication table with 40 different scalars, but the number of constants can be decreased by making a judicious choice of basis. This class of algebras clearly 
contains those division algebras with $\operatorname{Der} A=s u(3)$ where $A$ is not an irreducible $s u(3)$-module, and hence also the division algebras where $\operatorname{Der} A=$ compact $G_{2}$. It also contains the algebras with $\operatorname{Der} A=s u(2) \oplus s u(2)$, since in the notation of (5.3) and (5.4), the elements $\partial_{1}+\partial_{1}^{\prime}, \partial_{2}+\partial_{2}^{\prime}, \partial_{3}+\partial_{3}^{\prime}$ form a subalgebra of Der $A$ isomor. phic to $s u(2)$ under which $A$ has the decomposition $1+1+3+3$. We don't know whether the case when $\operatorname{Der} A=s u(3)$ and $A$ is an irreducible $s u(3)$-module is included in the present case, or whether there exist real division algebras with the decomposition $1+1+3+3$ where $\operatorname{Der} A$ is either just $s u(2)$ or $s u(2)+N$.

We turn briefly to the case where $A$ has the $s u(2)$-module decomposition $1+3+4$. The general multiplication table here can be written out using 21 constants. It is clear that those division algebras where $\operatorname{Der} A=s u(2) \oplus s u(2)$ or $\operatorname{Der} A=$ compact $G_{2}$ are included in this class. The division algebras with $\operatorname{Der} A=s u(3)$ and $A$ not an irreducible $s u(3)$-module are clearly not included in the $1+3+4$ case, but it is less clear whether the case when $\operatorname{Der} A=$ $s u(3)$ and $A$ is an irreducible $s u(3)$-module is included. We have not attempted to settle whether there are division algebras of this type with $\operatorname{Der} A=s u(2)$ or $\operatorname{Der} A=s u(2) \oplus N$ for the case $1+3+4$.

Our final case is when the $s u(2)$-module decomposition is $1+1+$ $1+1+4$. Again those division algebras where $\operatorname{Der} A=s u(2) \oplus s u(2)$ or Der $A=$ compact $G_{2}$ are included in this class. We don't know whether either type of division algebra with Der $A=s u(3)$ occurs here. For this case we will prove that there are division algebras with $\operatorname{Der} A=s u(2)$ and also with $\operatorname{Der} A=s u(2) \oplus N$.

Let $A$ be an algebra with basis $u, e_{1}, e_{2}, \cdots, e_{7}$ and multiplication as in the octonions except that the squares of the $e_{i}$ 's are not all equal. Specifically, products in $A$ are given by

$$
u^{2}=u, u e_{i}=e_{i}=e_{i} u, e_{i}^{2}=-\beta_{i} u, \quad \text { for } i=1, \cdots, 7
$$

$$
e_{i} e_{i+1}=e_{i+3}=-e_{i+1} e_{i}, e_{i+1} e_{i+3}=e_{i}=-e_{i+1} e_{i+3},
$$

$e_{i+3} e_{i}=e_{i+1}=-e_{i} e_{i+3}$, where the subscripts are taken modulo 7 ,

and where $\beta_{1}, \beta_{2}, \cdots, \beta_{7}$ are positive real numbers. We have shown [1, Theorem 20] that this algebra is a real division algebra, and we want to calculate its derivations for appropriate conditions on the $\beta$ 's. In particular, we shall establish

THEOREM 6.9. Let $A$ be the real division algebra defined by (6.8) and let $\beta_{3}=\beta_{5}=\beta_{6}=\beta_{7}$. If $\beta_{1}, \beta_{2}, \beta_{3}, \beta_{4}$ are distinct, then $\operatorname{Der} A=s u(2)$. If $\beta_{1}, \beta_{2}, \beta_{3}$ are distinct and $\beta_{2}=\beta_{4}$, then $\operatorname{Der} A=$ su $(2) \oplus N$ where $N$ is a 1-dimensional Lie algebra. 
Proof. Suppose that $\beta_{3}=\beta_{5}=\beta_{6}=\beta_{7}$, and define the linear transformations $\partial_{1}^{\prime}, \partial_{2}^{\prime}, \partial_{3}^{\prime}$, on $A$ by

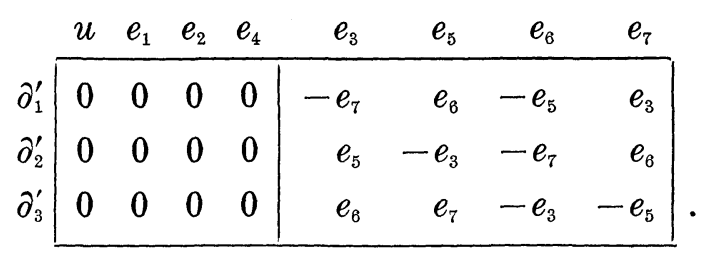

Comparing with (5.4), we see that (6.10) defines an irreducible module action of $s u(2)$ spanned by $\partial_{1}^{\prime}, \partial_{2}^{\prime}, \partial_{3}^{\prime}$ on the subspace $E_{1}=$ $\left\langle e_{3}, e_{5}, e_{6}, e_{7}\right\rangle$. By definition $s u(2)$ acts trivially on the subalgebra $E_{0}=\left\langle u, e_{1}, e_{2}, e_{4}\right\rangle$, and we need to verify that $\partial_{1}^{\prime}, \partial_{2}^{\prime}, \partial_{3}^{\prime}$ act like derivations on products of the form $E_{0} E_{1}, E_{1} E_{0}$, and $E_{1} E_{1}$.

Since we showed in $\S 5$ that $\partial_{1}^{\prime}, \partial_{2}^{\prime}, \partial_{3}^{\prime}$ are derivations of the octonions and since the present algebra is the same as the octonions except for the squares of the $e_{i}^{\prime} \mathrm{s}$, the maps $\partial_{1}^{\prime}, \partial_{2}^{\prime}, \partial_{3}^{\prime}$ will act as derivations on any product of basis vectors where the verification does not depend on calculating the square of an $e_{i}$. In particular, the $\partial_{i}^{\prime} \mathrm{s}$ act as derivations on all products of the form $E_{0} E_{1}$ or $E_{1} E_{0}$. For the remaining products-those of the type $E_{1} E_{1}$, one can verify directly using (6.10) and the fact that $\beta_{3}=\beta_{5}=\beta_{6}=\beta_{7}$ that each $\partial_{i}^{\prime}$ behaves as a derivation. Thus, $\partial_{1}^{\prime}, \partial_{2}^{\prime}, \partial_{3}^{\prime}$ are derivations and span a copy of $s u(2)$ in $\operatorname{Der} A$. need

In order to find out which other derivations of $A$ exist, we

LeMma 6.11. If $\partial$ is a derivation of the algebra $A$ defined by equations (6.8), then $\partial(u)=0$ and there exist real numbers $a_{i j}$ for $1 \leqq i, j \leqq 7$ such that $a_{i j}=-a_{j i}$ and $\partial\left(e_{i}\right)=\sum_{j=1}^{7} a_{i j} e_{j}$ for $1 \leqq i \leqq 7$. Futhermore, if $\beta_{i} \neq \beta_{j}$, then $a_{i j}=0$.

Proof. Since $u$ is the identity element of $A, \partial(u)=0$. If $\partial\left(e_{i}\right)=a_{i 0} u+\sum_{j=1}^{7} a_{i j} e_{j}$ for $a_{i 0}, a_{i j} \in \boldsymbol{R}$, we see from

$$
0=\partial\left(e_{i}^{2}\right)=\partial\left(e_{i}\right) e_{i}+e_{i} \partial\left(e_{i}\right)=2 a_{i 0} e_{i}-2 \beta_{i} a_{i i} u
$$

that $a_{i 0}=0=a_{i i}$ for $1 \leqq i \leqq 7$. For fixed $i \neq j$, there exists $k$ such that either $e_{i}=e_{j} e_{k}$ or $e_{i}=e_{k} e_{j}$. In the former case, the $e_{j}$-component of

$$
\partial\left(e_{i}\right)=\partial\left(e_{j} e_{k}\right)=\partial\left(e_{j}\right) e_{k}+e_{j} \partial\left(e_{k}\right)=\sum_{l} a_{j l} e_{l} e_{k}+\sum_{l} a_{k l} e_{j} e_{l}
$$




$$
a_{i j}=-a_{j i},
$$

since $e_{j} e_{l}$ is never a multiple of $e_{j}$ and since $e_{l} e_{k}$ is a multiple of $e_{j}$ exactly when $l=i$, in which case $e_{i} e_{k}=-e_{j}$. If $e_{i}=e_{k} e_{j}$, then (6.12) also holds by the identical argument with left and right interchanged. If $\beta_{i} \neq \beta_{j}$, then

$$
0=\partial\left(e_{i} e_{j}+e_{j} e_{i}\right)=\partial\left(e_{i}\right) e_{j}+e_{i} \partial\left(e_{j}\right)+\partial\left(e_{j}\right) e_{i}+e_{j} \partial\left(e_{i}\right),
$$

and the $u$-component of this is

$$
0=2 a_{i j} e_{j}^{2}+2 a_{j i} e_{i}^{2}=2 a_{i j}\left(e_{j}^{2}-e_{i}^{2}\right)=2 a_{i j}\left(\beta_{i}-\beta_{j}\right) u,
$$

which implies that $a_{i j}=0$.

Returning to the proof of Theorem 6.9, we suppose first that $\beta_{1}, \beta_{2}, \beta_{3}, \beta_{4}$ are distinct. Then, for any $\partial \in \operatorname{Der} A$, we see from Lemma 6.11 that $a_{i j}=0$ unless $i$ and $j$ are both in the set $\{3,5,6,7\}$, giving $\partial\left(E_{0}\right)=0$ and $\partial\left(E_{1}\right) \cong E_{1}$. Hence $\partial\left(e_{3}\right)=b_{5} e_{5}+b_{6} e_{6}+b_{7} e_{7}$ for some $b_{5}, b_{6}, b_{7} \in \boldsymbol{R}$, and $\partial^{\prime}=\partial-b_{5} \partial_{2}^{\prime}-b_{8} \partial_{3}^{\prime}+b_{7} \partial_{1}^{\prime}$ has the property that $\partial^{\prime}\left(e_{3}\right)=0$. It follows that

$$
\begin{aligned}
& \partial^{\prime}\left(e_{5}\right)=\partial^{\prime}\left(e_{2} e_{3}\right)=\partial^{\prime}\left(e_{2}\right) e_{3}+e_{2} \partial^{\prime}\left(e_{3}\right)=0, \\
& \partial^{\prime}\left(e_{6}\right)=\partial^{\prime}\left(e_{3} e_{4}\right)=\partial^{\prime}\left(e_{3}\right) e_{4}+e_{3} \partial^{\prime}\left(e_{4}\right)=0, \\
& \partial^{\prime}\left(e_{7}\right)=\partial^{\prime}\left(e_{1} e_{3}\right)=\partial^{\prime}\left(e_{1}\right) e_{3}+e_{1} \partial^{\prime}\left(e_{3}\right)=0,
\end{aligned}
$$

giving $\partial^{\prime}=0$. Thus, $\partial=b_{5} \partial_{2}^{\prime}+b_{6} \partial_{3}^{\prime}-b_{7} \partial_{1}^{\prime} \in s u(2)$, and Der $A=s u(2)$.

Finally, suppose that $\beta_{1}, \beta_{2}, \beta_{3}$ are distinct and that $\beta_{2}=\beta_{4}$. If $\partial \in \operatorname{Der} A$, then Lemma 6.11 implies that

$$
\partial\left(e_{1}\right)=0, \partial\left(e_{2}\right)=a_{24} e_{4}, \partial\left(e_{4}\right)=-a_{24} e_{2}, \partial\left(E_{1}\right) \subseteq E_{1} .
$$

If $a_{24}=0$, the argument of the last paragraph shows that $\partial \in s u(2)$, implying that $\operatorname{dim} \operatorname{Der} A \leqq \operatorname{dim} s u(2)+1=4$. Thus, in order to prove that $\operatorname{Der} A=s u(2)+N$, it is sufficient to show that $\operatorname{Der} A$ contains a nonzero derivation $\partial_{1}$ which commutes with $\partial_{1}^{\prime}, \partial_{2}^{\prime}, \partial_{3}^{\prime}$. We claim that if $\partial_{1}$ is defined by

$$
\begin{aligned}
& \partial_{1}(u)=0, \partial_{1}\left(e_{1}\right)=0, \partial_{1}\left(e_{2}\right)=2 e_{4}, \partial_{1}\left(e_{4}\right)=-2 e_{2}, \\
& \partial_{1}\left(e_{3}\right)=-e_{7}, \partial_{1}\left(e_{5}\right)=-e_{6}, \partial_{1}\left(e_{6}\right)=e_{5}, \partial_{1}\left(e_{7}\right)=e_{3},
\end{aligned}
$$

then $\partial_{1}$ is a derivation of $A$ commuting with $\partial_{1}^{\prime}, \partial_{2}^{\prime}, \partial_{3}^{\prime}$. We saw in $\S 5$ that $\partial_{1}$ is a derivation of the octonions commuting with $\partial_{1}^{\prime}, \partial_{2}^{\prime}, \partial_{3}^{\prime}$ (see (5.4)). Thus $\partial_{1}$ must also commute here with $\partial_{1}^{\prime}, \partial_{2}^{\prime}, \partial_{3}^{\prime}$, and $\partial_{1}$ must act as a derivation on any product of basis vectors, since in those cases where the calculation involves squaring on $e_{i}$, the two 
$\beta$ 's involved will be equal. Therefore, $\partial_{1}$ is a derivation here also.

7. The case when $\operatorname{Der} A$ is abelian. We consider next the case when Der $A$ is abelian of dimension 2. By [1, Corollary 16], $\operatorname{dim} A=8$ and there is a basis $\partial_{1}^{\prime}, \partial_{2}^{\prime}$ of Der $A$ such that $\partial_{1}^{\prime}$ and $\partial_{2}^{\prime}$ are diagonal relative to a suitable choice of basis of $A_{c}$ and are of the form

$$
\begin{aligned}
& \partial_{1}^{\prime} \longleftrightarrow \operatorname{diag}\{0,0, \alpha i,-\alpha i, \beta i,-\beta i,(\alpha+\beta) i,-(\alpha+\beta) i\} \\
& \partial_{2}^{\prime} \longleftrightarrow \operatorname{diag}\{0,0,0,0, \gamma i,-\gamma i, \gamma i,-\gamma i\}
\end{aligned}
$$

for nonzero real numbers $\alpha, \beta, \gamma$. Then $\partial_{1}=(1 / \alpha) \partial_{1}^{\prime}-(\beta / \alpha \gamma) \partial_{2}^{\prime}$ and $\partial_{2}=(1 / \gamma) \partial_{2}^{\prime}$ are also a basis for $\operatorname{Der} A$ and

$$
\begin{aligned}
& \partial_{1} \longleftrightarrow \operatorname{diag}\{0,0, i,-i, 0,0, i,-i\} \\
& \partial_{2} \longleftrightarrow \operatorname{diag}\{0,0,0,0, i,-i, i,-i\} .
\end{aligned}
$$

Hence there must exist a basis $u_{1}, u_{2}, x_{1}, x_{2}, y_{1}, y_{2}, z_{1}, z_{2}$ of $A$ such that $\partial_{1}$ and $\partial_{2}$ are given by

$$
\begin{aligned}
& \partial_{1}\left(u_{1}\right)=0=\partial_{1}\left(u_{2}\right), \partial_{1}\left(x_{1}\right)=x_{2}, \partial_{1}\left(x_{2}\right)=-x_{1}, \partial_{1}\left(y_{1}\right)=0=\partial_{1}\left(y_{2}\right), \\
& \partial_{1}\left(z_{1}\right)=z_{2}, \partial_{1}\left(z_{2}\right)=-z_{1}, \partial_{2}\left(u_{1}\right)=0=\partial_{2}\left(u_{2}\right), \partial_{2}\left(x_{1}\right)=0=\partial_{2}\left(x_{2}\right) \\
& \partial_{2}\left(y_{1}\right)=y_{2}, \partial_{2}\left(y_{2}\right)=-y_{1}, \partial_{2}\left(z_{1}\right)=z_{2}, \partial_{2}\left(z_{2}\right)=-z_{1} .
\end{aligned}
$$

Defining the subspaces $U, X, Y, Z$ of $A$ by

$$
U=\left\langle u_{1}, u_{2}\right\rangle, X=\left\langle x_{1}, x_{2}\right\rangle, Y=\left\langle y_{1}, y_{2}\right\rangle, Z=\left\langle z_{1}, z_{2}\right\rangle,
$$

we will show first that the product of any two of these spaces is contained in one of the subspaces. Specifically, we will prove

LEMmA 7.3. The products of the spaces $U, X, Y, Z$ are given by the table

\begin{tabular}{c|cccc}
\multicolumn{1}{c}{} & $U$ & $X$ & $Y$ & $Z$ \\
\hline$U$ & $U$ & $X$ & $Y$ & $Z$ \\
$X$ & $X$ & $U$ & $Z$ & $Y$ \\
$Y$ & $Y$ & $Z$ & $U$ & $X$ \\
$Z$ & $Z$ & $Y$ & $X$ & $U$ \\
\hline
\end{tabular}

Proof. Since $U$ is the kernel of $\operatorname{Der} A$, we have $U^{2} \cong U$. If 
$u \in U$ and $x \in X$, then $u, x \in \operatorname{ker} \partial_{2}$, so $u x \in \operatorname{ker} \partial_{2}=U+X$. On the other hand, $x$ is the image of some element $x^{\prime} \in X$ under $\partial_{1}$, and so $u x=u \partial_{1}\left(x^{\prime}\right)=\partial_{1}\left(u x^{\prime}\right)$ is also in the image of $\partial_{1}$ which is $X+Z$. Thus $u x \in(U+X) \cap(X+Z)=X$. Similarly, for $u \in U$ and $y \in Y$, we have

$$
u y \in\left(\operatorname{ker} \partial_{1}\right) \cap\left(\operatorname{Im} \partial_{2}\right)=(U+Y) \cap(Y+Z)=Y .
$$

For $u \in U$ and $z \in Z$, we define $\partial_{3}=\partial_{1}-\partial_{2}$ and note that

$$
u z \in\left(\operatorname{ker} \partial_{3}\right) \cap\left(\operatorname{Im} \partial_{1}\right)=(U+Z) \cap(X+Z)=Z .
$$

The same calculations show that $X U \subseteq X, Y U \subseteq Y$, and $Z U \subseteq Z$. Also, if $x \in X, y \in Y, z \in Z$, we obtain

$$
\begin{aligned}
& x y, y x \in\left(\operatorname{Im} \partial_{1}\right) \cap\left(\operatorname{Im} \partial_{2}\right)=(X+Z) \cap(Y+Z)=Z, \\
& x z, z x \in\left(\operatorname{Im} \partial_{2}\right) \cap\left(\operatorname{Im} \partial_{3}\right)=(Y+Z) \cap(X+Y)=Y, \\
& y z, z y \in\left(\operatorname{Im} \partial_{1}\right) \cap\left(\operatorname{Im} \partial_{3}\right)=(X+Z) \cap(X+Y)=X .
\end{aligned}
$$

Finally, if $x, x^{\prime} \in X$, then we calculate that

$$
\begin{gathered}
\partial_{1}\left(x x^{\prime}\right)=\partial_{1}(x) x^{\prime}+x \partial_{1}\left(x^{\prime}\right), \partial_{1}^{2}\left(x x^{\prime}\right)=-x x^{\prime}+2 \partial_{1}(x) \partial_{1}\left(x^{\prime}\right)-x x^{\prime}, \\
\partial_{1}^{3}\left(x x^{\prime}\right)=-2 \partial_{1}(x) x^{\prime}-2 x \partial_{1}\left(x^{\prime}\right)-2 x \partial_{1}\left(x^{\prime}\right)-2 \partial_{1}(x) x^{\prime} .
\end{gathered}
$$

But $\partial_{1}^{2}$ acting on $\operatorname{Im} \partial_{1}$ has the effect of multiplying by -1 , and so

$$
\begin{aligned}
0 & =\partial_{1}\left(x x^{\prime}\right)+\partial_{1}^{3}\left(x x^{\prime}\right)=\partial_{1}(x) x^{\prime}+x \partial_{1}\left(x^{\prime}\right)-4 \partial_{1}(x) x^{\prime}-4 x \partial_{1}\left(x^{\prime}\right) \\
& =-3 \partial_{1}\left(x x^{\prime}\right)
\end{aligned}
$$

showing that $x x^{\prime} \in \operatorname{ker} \partial_{1}$. Since $x, x^{\prime} \in \operatorname{ker} \partial_{2}$, so is $x x^{\prime}$, and

$$
x x^{\prime} \in\left(\operatorname{ker} \partial_{1}\right) \cap\left(\operatorname{ker} \partial_{2}\right)=(U+Y) \cap(U+X)=U .
$$

By an identical argument, we obtain $Y^{2} \subseteq U$ and $Z^{2} \subseteq U$.

The existence of the two commuting derivations $\partial_{1}, \partial_{2}$ not only gives the block multiplication of Lemma 7.3 but also imposes some conditions on how the elements of these different blocks multiply. In particular, we have

THEOREM 7.5. If $A$ is a real division algebra which has two linearly independent commuting derivations, then $A$ has a basis $u_{1}, u_{2}, x_{1}, x_{2}, y_{1}, y_{2}, z_{1}, z_{2}$ for which the multiplication table (7.6) holds. 


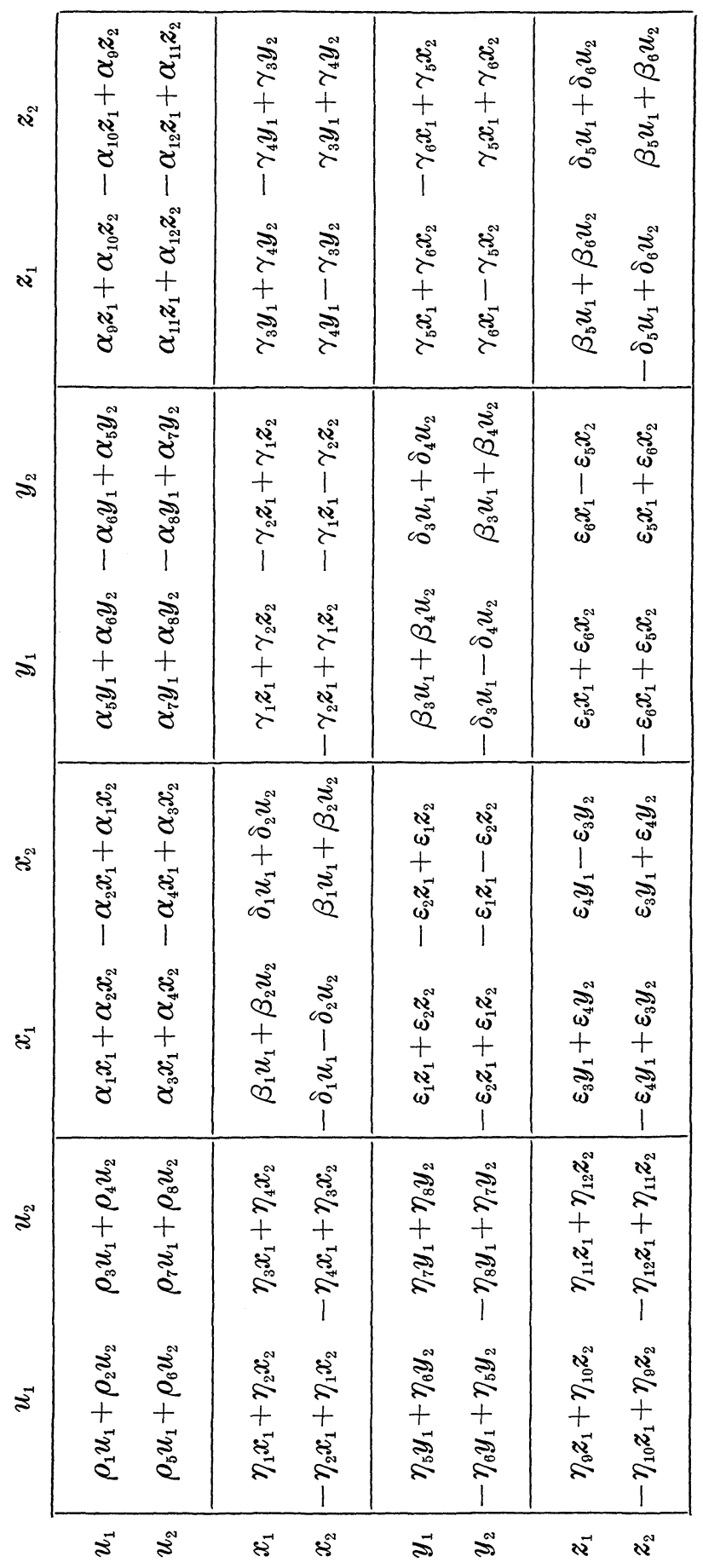


Proof. From (7.4) we know that $x_{1} y_{1} \in Z$, say $x_{1} y_{1}=\gamma_{1} z_{1}+\gamma_{2} z_{2}$. Applying $\partial_{1}$ and $\partial_{2}$ respectively to this relation and using (7.2), we obtain

$$
\begin{aligned}
& x_{2} y_{1}=\partial_{1}\left(x_{1}\right) y_{1}=\partial_{1}\left(x_{1} y_{1}\right)=\partial_{1}\left(\gamma_{1} z_{1}+\gamma_{2} z_{2}\right)=\gamma_{1} z_{2}-\gamma_{2} z_{1} \\
& x_{1} y_{2}=x_{1} \partial_{2}\left(y_{1}\right)=\partial_{2}\left(x_{1} y_{1}\right)=\gamma_{1} z_{2}-\gamma_{2} z_{1} .
\end{aligned}
$$

And applying $\partial_{1}$ to the last relation gives $x_{2} y_{2}=\partial_{1}\left(x_{1} y_{2}\right)=-\gamma_{1} z_{1}-$ $\gamma_{2} z_{2}$. Similarly, there exist $\gamma_{3}, \gamma_{4}, \gamma_{5}, \gamma_{6} \in \boldsymbol{R}$ such that $x_{1} z_{1}=\gamma_{3} y_{1}+$ $\gamma_{4} y_{2}$ and $y_{1} z_{1}=\gamma_{5} x_{1}+\gamma_{6} x_{2}$, and the application of $\partial_{1}, \partial_{2}, \partial_{3}=\partial_{1}-\partial_{2}$ to these equations gives the remaining products of the form $X Z$ and $Y Z$. The products of the form $Y X, Z X$, and $Z Y$ follow by leftright symmetry.

Next, choosing $\alpha_{1}, \alpha_{2}, \alpha_{3}, \alpha_{4} \in \boldsymbol{R}$ such that $u_{1} x_{1}=\alpha_{1} x_{1}+\alpha_{2} x_{2}$ and $u_{2} x_{1}=\alpha_{3} x_{1}+\alpha_{4} x_{2}$, we get

$$
u_{1} x_{2}=u_{1} \partial_{1}\left(x_{1}\right)=\partial_{1}\left(u_{1} x_{1}\right)=\alpha_{1} x_{2}-\alpha_{2} x_{1}, u_{2} x_{2}=\partial_{1}\left(u_{2} x_{1}\right)=\alpha_{3} x_{2}-\alpha_{4} x_{1} .
$$

By identical arguments, we obtain all the entries in (7.4) of the forms $U X, U Y, U Z, X U, Y U$, and $Z U$. Choosing $\beta_{1}, \beta_{2}, \delta_{1}, \delta_{2} \in \boldsymbol{R}$ with $x_{1}^{2}=\beta_{1} u_{1}+\beta_{2} u_{2}$ and $x_{1} x_{2}=\delta_{1} u_{1}+\delta_{2} u_{2}$, we have the relations

$$
\begin{aligned}
& 0=\partial_{1}\left(x_{1}^{2}\right)=\partial_{1}\left(x_{1}\right) x_{1}+x_{1} \partial_{1}\left(x_{2}\right)=x_{2} x_{1}+x_{1} x_{2}, \\
& 0=\partial_{1}\left(x_{1} x_{2}\right)=\partial_{1}\left(x_{1}\right) x_{2}+x_{1} \partial_{1}\left(x_{2}\right)=x_{2}^{2}-x_{1}^{2}
\end{aligned}
$$

which give us $x_{2}^{2}$ and $x_{2} x_{1}$. The entries in (7.4) of the form $Y^{2}$ and $Z^{2}$ are found in the same way. Finally, the derivations $\partial_{1}$ and $\partial_{2}$ impose no restrictions at all on the subspace $U$, so the constants have to be all different here.

As our final result, we establish

THEOREM 7.7. Let $A$ be the modified octonion algebra defined by (6.8). Then

(i) if $\beta_{1}, \cdots, \beta_{7}$ are all distinct, $\operatorname{Der} A=0$.

(ii) if $\beta_{1}, \beta_{2}, \beta_{3}, \beta_{4}, \beta_{6}$ are distinct, $\beta_{3}=\beta_{5}$, and $\beta_{6}=\beta_{7}$, then $\operatorname{dim} \operatorname{Der} A=1$.

(iii) if $\beta_{1}, \beta_{2}, \beta_{3}, \beta_{6}$ are distinct, $\beta_{1}=\beta_{4}, \beta_{3}=\beta_{5}$ and $\beta_{6}=\beta_{7}$, then $\operatorname{dim} \operatorname{Der} A=2$ and $\operatorname{Der} A$ is abelian.

Proof. If $\beta_{1}, \cdots, \beta_{7}$ are all distinct, it is immediate from Lemma 6.11 that $A$ cannot have any nonzero derivations, giving part (i). If the hypotheses of part (ii) of Theorem 7.7 hold and if $\partial \in \operatorname{Der} A$, then Lemma 6.11 implies that 


$$
\begin{gathered}
\partial\left(e_{3}\right)=a_{35} e_{5}, \partial\left(e_{5}\right)=-a_{35} e_{3}, \partial\left(e_{6}\right)=a_{67} e_{7}, \partial\left(e_{7}\right)=-a_{67} e_{6}, \\
\partial(u)=0=\partial\left(e_{1}\right), \partial\left(e_{2}\right)=0=\partial\left(e_{4}\right),
\end{gathered}
$$

for some $a_{35}, a_{67} \in \boldsymbol{R}$. From

$$
a_{35} e_{5}=\partial\left(e_{3}\right)=\partial\left(e_{7} e_{1}\right)=\partial\left(e_{7}\right) e_{1}=-a_{67} e_{6} e_{1}=-a_{67} e_{5}
$$

we get $a_{35}=-a_{67}$. Thus, Der $A$ is at most 1-dimensional. To show that $\operatorname{dim} \operatorname{Der} A=1$, it is sufficient to verify that the special case of (7.8) with $a_{35}=1$ and $a_{67}=-1$ is a derivation of $A$. But this linear transformation was shown to be a derivation of the octonions in $\S 5$ (under the correspondence $e_{3} \leftrightarrow y_{1}, e_{5} \leftrightarrow y_{2}, e_{6} \leftrightarrow y_{3}, e_{7} \leftrightarrow y_{4}, \partial$ corresponds to $\partial_{2}^{\prime}$ in (5.4)), and so $\partial$ will act as a derivation on any product of basis vectors where the verification does not depend on calculating the square of an $e_{i}$. Since $\beta_{3}=\beta_{5}$ and $\beta_{6}=\beta_{7}$ in the case we are considering, it is clear from (7.8) that $\partial$ will act as a derivation even in those cases where the verification depends on calculating the square of an $e_{i}$.

Finally, suppose that the hypotheses of part (iii) of Theorem 7.7 hold. Then Lemma 6.11 shows that any $\partial \in \operatorname{Der} A$ has the form

$$
\begin{aligned}
& \partial(u)=0=\partial\left(e_{2}\right), \partial\left(e_{1}\right)=a_{14} e_{4}, \partial\left(e_{4}\right)=-a_{14} e_{1}, \\
& \partial\left(e_{3}\right)=a_{35} e_{5}, \partial\left(e_{5}\right)=-a_{35} e_{3}, \partial\left(e_{6}\right)=a_{67} e_{7}, \partial\left(e_{7}\right)=-a_{67} a_{6},
\end{aligned}
$$

for some $a_{14}, a_{35}, a_{87} \in \boldsymbol{R}$. Since

$$
\begin{aligned}
a_{67} e_{7}=\partial\left(e_{6}\right) & =\partial\left(e_{3} e_{4}\right)=\partial\left(e_{3}\right) e_{4}+e_{3} \partial\left(e_{4}\right)=a_{35} e_{5} e_{4}-a_{14} e_{3} e_{1} \\
& =\left(a_{35}+a_{14}\right) e_{7},
\end{aligned}
$$

we see that $\operatorname{dim} \operatorname{Der} A \leqq 2$. It suffices to show that the special cases of (7.9) defined by the table

$$
\begin{array}{c|cccccccc|} 
& \multicolumn{1}{c}{u} & e_{1} & e_{2} & e_{4} & e_{3} & e_{5} & e_{8} & e_{7} \\
\partial_{2} & 0 & -2 e_{4} & 0 & 2 e_{1} & -e_{5} & e_{3} & -e_{7} & e_{6} \\
\partial_{2}^{\prime} & 0 & 0 & 0 & 0 & e_{5} & -e_{3} & -e_{7} & e_{6} \\
\hline
\end{array}
$$

are both derivations of $A$. Again $\partial_{2}$ and $\partial_{2}^{\prime}$ were shown in $\S 5$ to be derivations of the octonions (see (5.4)), and as we argued in the last case, $\partial_{2}$ and $\partial_{2}^{\prime}$ must be derivations here because $\beta_{1}=\beta_{4}, \beta_{3}=\beta_{5}$, and $\beta_{8}=\beta_{7}$.

REMARK. If $A$ is a finite-dimensional real algebra with $L=$ Der $A$ as its derivation algebra, then the connected Lie group $G$ corresponding to the Lie algebra $L$ acts as a group of automorphisms on $A$. Furthermore, $G$ necessarily has finite index in Aut $A$, 
the group of all automorphisms of $A$. One might ask in the case of a real division algebra whether $G$ can be properly contained in Aut $A$, and we shall give an example to show that this can happen. In the algebra $A$ defined by (6.8) with all $\beta$ 's distinct, we have shown that Der $A=0$ and hence $G=1$. On the other hand, this algebra has 8 automorphisms, as one sees by noting that for any choice of $\varepsilon_{1}, \varepsilon_{2}, \varepsilon_{3} \in\{1,-1\}$ the map

$$
\begin{aligned}
a_{0} u & +\sum a_{i} e_{i} \longrightarrow a_{0} u+a_{1} \varepsilon_{1} e_{1}+a_{2} \varepsilon_{2} e_{2}+a_{3} \varepsilon_{3} e_{3}+a_{4} \varepsilon_{1} \varepsilon_{2} e_{4} \\
& +a_{5} \varepsilon_{2} \varepsilon_{3} e_{5}+a_{8} \varepsilon_{1} \varepsilon_{2} \varepsilon_{3} e_{6}+a_{7} \varepsilon_{1} \varepsilon_{3} e_{7}
\end{aligned}
$$

is an automorphism of $A$.

\section{REFERENCES}

1. G. M. Benkart and J. M. Osborn, The derivation algebra of a real division algebra, to appear, in Amer. J.

2. M. Gunaydin and F. Gursey, Quark structure and octonions, J. Math. Phys., 14 (1973), 1651-1667.

3. I. N. Herstein, Topics in Algebra, Blaisdell, 1964.

4. J. E. Humphreys, Introduction to Lie Algebras and Representation Theory, Springer Verlag, 1972.

5. N. Jacobson, Composition algebras and their automorphisms, Rend. Circ. Mat. Palermo, (2), 7 (1958), 55-80.

6. Lie Algebras, Wiley Interscience, 1962.

7. — Exceptional Lie Algebras, Marcel Dekker, 1971.

8. - S. Okubo, Pseudo-quaternion and pseudo-octonion algebras, Hadronic Journal, 1 (1978), 1250-1278.

9. R. D. Schafer, Introduction to Nonassociative Algebras, Academic Press, 1966.

Received August 20, 1979. Partially supported by N.S.F. grant \#MCS77-01724.

UNIVERSITY OF WISCONSIN

MADISON, WI 53706 


\section{PACIFIC JOURNAL OF MATHEMATICS}

\section{EDITORS}

DoNALD BABBITT (Managing Editor)

University of California

Los Angeles, CA 90024

Hugo Rossi

University of Utah

Salt Lake City, UT 84112

C. C. MOORE and ANDREw OGG

University of California

Berkeley, CA 94720
J. DugundJI

Department of Mathematics

University of Southern California

Los Angeles, CA 90007

R. FinN and J. Milgram

Stanford University

Stanford, CA 94305

ASSOCIATE EDITORS
R. ARENS
E. F. BECKENBACH
B. H. NeumanN
F. WOLF
K. YoshidA

\section{SUPPORTING INSTITUTIONS}

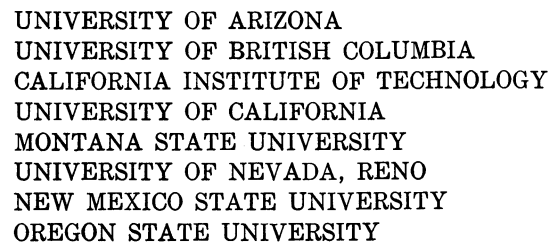

UNIVERSITY OF ARIZONA

UNIVERSITY OF BRITISH COLUMBIA

CALIFORNIA INSTITUTE OF TECHNOLOGY

UNIVERSITY OF CALIFORNIA

MONTANA STATE UNIVERSITY

UNIVERSITY OF NEVADA, RENO

NEW MEXICO STATE UNIVERSITY OREGON STATE UNIVERSITY

\author{
UNIVERSITY OF OREGON \\ UNIVERSITY OF SOUTHERN CALIFORNIA \\ STANFORD UNIVERSITY \\ UNIVERSITY OF HAWAII \\ UNIVERSITY OF TOKYO \\ UNIVERSITY OF UTAH \\ WASHINGTON STATE UNIVERSITY \\ UNIVERSITY OF WASHINGTON
}

The Supporting Institutions listed above contribute to the cost of publication of this Journal, but they are not owners or publishers and have no responsibility for its content or policies.

Mathematical papers intended for publication in the Pacific Journal of Mathematics should be in typed form or offset-reproduced, (not dittoed), double spaced with large margins. Please do not use built up fractions in the text of the manuscript. However, you may use them in the displayed equations. Underline Greek letters in red, German in green, and script in blue. The first paragraph or two must be capable of being used separately as a synopsis of the entire paper. Please propose a heading for the odd numbered pages of less than 35 characters. Manuscripts, in triplicate, may be sent to any one of the editors. Please classify according to the scheme of Math. Reviews, Index to Vol. 39. Supply name and address of author to whom proofs should be sent. All other communications should be addressed to the managing editor, or Elaine Barth, University of California, Los Angeles, California, 90024.

50 reprints to each author are provided free for each article, only if page charges have been substantially paid. Additional copies may be obtained at cost in multiples of 50 .

The Pacific Journal of Mathematics is issued monthly as of January 1966. Regular subscription rate: $\$ 102.00$ a year (6 Vols., 12 issues). Special rate: $\$ 51.00$ a year to individual members of supporting institutions.

Subscriptions, orders for numbers issued in the last three calendar years, and changes of address shoud be sent to Pacific Journal of Mathematics, P.O. Box 969, Carmel Valley, CA 93924, U.S.A. Old back numbers obtainable from Kraus Periodicals Co., Route 100, Millwood, NY 10546.

\footnotetext{
PUBLISHED BY PACIFIC JOURNAL OF MATHEMATICS, A NON-PROFIT CORPORATION

Printed at Kokusai Bunken Insatsusha (International Academic Printing Co., Ltd.). 8-8, 3-chome, Takadanobaba, Shinjuku-ku, Tokyo 160, Japan.
} 


\section{Pacific Journal of Mathematics}

\section{Vol. 96, No. 2 December, 1981}

Gerald A. Beer, A natural topology for upper semicontinuous functions and

a Baire category dual for convergence in measure $\ldots \ldots \ldots \ldots \ldots \ldots 251$

Georgia Benkart and J. Marshall Osborn, An investigation of real

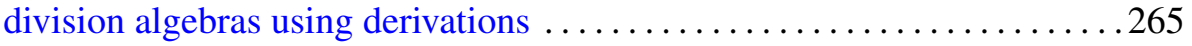

Donald Ian Cartwright and John R. McMullen, A structural criterion for the existence of infinite Sidon sets ........................ 301

Philip Hanlon, The fixed-point partition lattices $\ldots \ldots \ldots \ldots \ldots \ldots \ldots \ldots$

Eric Hayashi, The spectral density of a strongly mixing stationary Gaussian process

Chung-Wu Ho and Charles E. Morris, Jr., A graph-theoretic proof of

Sharkovsky's theorem on the periodic points of continuous functions . ...361

Sara Hurvitz, The automorphism groups of spaces and fibrations ....... 371

Atsushi Inoue, Schoichi Ota and Jun Tomiyama, Derivations of operator algebras into spaces of unbounded operators . .................. 389

Wolfgang B. Jurkat and Gary Sampson, On weak restricted estimates and endpoint problems for convolutions with oscillating kernels. I ........ 405

Georgios Koumoullis, Some topological properties of spaces of

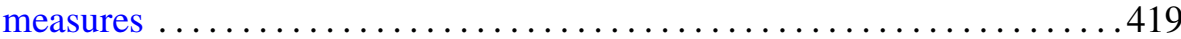

Wen Hsiung Lin, Algebraic Kahn-Priddy theorem ................. 435

Michael John McAsey, Invariant subspaces of nonselfadjoint crossed

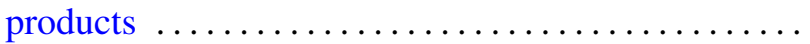

Justin Peters, Entropy of automorphisms on L.C.A. groups

Saburou Saitoh, A characterization of the adjoint $L$-kernel of Szegó type 